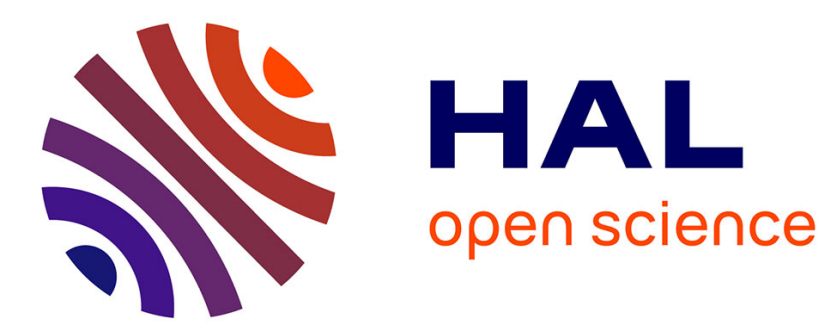

\title{
A survey and new results on Banach algebras of ultrametric continuous functions
}

Monique Chicourrat, Bertin Diarra, Alain Escassut

\section{To cite this version:}

Monique Chicourrat, Bertin Diarra, Alain Escassut. A survey and new results on Banach algebras of ultrametric continuous functions. p-Adic Numbers, Ultrametric Analysis and Applications, In press, 12 (3), pp.185-202. hal-02882656

\section{HAL Id: hal-02882656 https://hal.science/hal-02882656}

Submitted on 27 Jun 2020

HAL is a multi-disciplinary open access archive for the deposit and dissemination of scientific research documents, whether they are published or not. The documents may come from teaching and research institutions in France or abroad, or from public or private research centers.
L'archive ouverte pluridisciplinaire HAL, est destinée au dépôt et à la diffusion de documents scientifiques de niveau recherche, publiés ou non, émanant des établissements d'enseignement et de recherche français ou étrangers, des laboratoires publics ou privés. 


\title{
A survey and new results
}

\section{on Banach algebras of ultrametric continuous functions}

\author{
by Monique Chicourrat, Bertin Diarra and Alain Escassut
}

\begin{abstract}
Let $\mathbb{I K}$ be an ultrametric complete valued field and $\mathbb{E}$ be an ultrametric space. We examine some Banach algebras $S$ of bounded continuous functions from $\mathbb{E}$ to $\mathbb{K}$ with the use of ultrafilters, particularly the relation of stickness. We recall and deepen results obtained in a previous paper by N. Maïnetti and the third author concerning the whole algebra $\mathcal{A}$ of all bounded continuous functions from $\mathbb{E}$ to $\mathbb{K}$. We show that every maximal ideal of finite codimension of $\mathcal{A}$ is of codimension 1. Moreover, that property holds for every algebra $S$, provided $\mathbb{K}$ is perfect. If $S$ admits the uniform norm on $\mathbb{E}$ as its spectral norm, then every maximal ideal is the kernel of only one multiplicative semi-norm, the Shilov boundary is equal to the whole multiplicative spectrum and the Banaschewski compactifiaction of $\mathbb{E}$ is homeomorphic to the multiplicative spectrum of $S$.
\end{abstract}

\section{Introduction and basic results in topology}

Let $\mathbb{K}$ be an ultrametric complete valued field and $\mathbb{E}$ be an ultrametric space. It is well known that the set of maximal ideals of a Banach $\mathbb{K}$-algebra is not sufficient to describe its spectral properties: we have to consider the set of continuous multiplicative semi-norms often called the multiplicative spectrum [2], [8], [11], [12], [14], [16]. In this paper, we generalize some of the results obtained in [15] to some Banach algebras of continuous bounded functions that we call semi-admissible algebras which concern maximal ideals, multiplicative spectrum, Shilov boundary and the Stone space of the Boolean ring of the clopen subsets of $\mathbb{E}$. The relation of stickness on ultrafilters, already considered in [14], here plays an important role.

Notations and definitions: Let $\mathbb{I K}$ be a complete valued field with respect to an ultrametric absolute value $|$.$| and let \mathbb{E}$ denote a metric space whose distance $\delta$ is ultrametric. Given $a \in \mathbb{E}$ and $r>0$, we denote by $d_{\mathbb{E}}\left(a, r^{-}\right)$the open ball $\{x \in \mathbb{E} \mid \delta(a, x)<r\}$ and particularly in $\mathbb{K}$ we denote by $d\left(a, r^{-}\right)$the open disk $\{x \in \mathbb{K}|| x-a \mid<r\}$. In the same way, in $\mathbb{E}$, we denote by $d_{\mathbb{E}}(a, r)$ the closed ball $\{x \in \mathbb{E} \mid \delta(a, x) \leq r\}$ and we denote by $d(a, r)$ the closed disk $\{x \in \mathbb{K}|| x-a \mid \leq r\}$. Moreover, in $\mathbb{K}$, we denote by $C(a, r)$ the set $\{x \in \mathbb{K}|| x-a \mid=r\}$.

We denote by $|\cdot|_{\infty}$ the Archimedean absolute value of $\mathbb{R}$.

Given a subset $H$ of $\mathbb{E}$, we denote by $\bar{H}$ the closure of $H$ in $\mathbb{E}$ and the function $u$ defined on $\mathbb{E}$ by $u(x)=1$ if $x \in H$ and $u(x)=0$ otherwise is called the characteristic function of $H$.

We will denote by $\mathbb{B}(\mathbb{E})$ the Boolean ring of clopen subsets of $\mathbb{E}$ with respect to the two classical laws $\Delta$ and $\cap$. 
Let us recall this obvious lemma:

Lemma 1.1: Let $F$ be a subset of $\mathbb{E}$ and let $u$ be its characteristic function. Then $u$ is continuous if and only if $F$ is clopen.

The following lemma is also clear since each ball of $\mathbb{K}$ is clopen.

Lemma 1.2: Let $f$ be a continuous function from $\mathbb{E}$ to $\mathbb{K}$ and let $M>0$. Given $M>0$, the sets $E_{1}=\{x \in \mathbb{E}|f(x)| \geq M\}$ and $E_{2}=\{x \in \mathbb{E}|f(x)| \leq M\}$ are clopen.

Corollary 1.2.a: Let $f$ be a continuous function from $\mathbb{E}$ to $\mathbb{K}$, let $M>0$ and let $h>0$. Then $\left\{x \in \mathbb{E}|||f(x)|-\left.M\right|_{\infty} \leq h\right\}$ is clopen.

Given a normed $\mathbb{K}$-algebra whose norm is $\|$. $\|$, we denote by $\|$. $\|_{s p}$ the spectral semi-norm that is associated and defined as $\|f\|_{s p}=\lim _{n \rightarrow+\infty}\left(\left\|f^{n}\right\|\right)^{\frac{1}{n}}$.

The Banach $\mathbb{K}$-algebra $\mathcal{A}$ of all bounded continuous functions from $\mathbb{E}$ to $\mathbb{K}$ is provided with the norm $\|$. $\|_{0}$ that we will call the uniform norm and which is defined as $\|f\|_{0}=$ $\sup \{|f(x)| \mid x \in \mathbb{E}\}$.

Recall the following:

Proposition 1.3: $\quad$ Let $T$ be a commutative unital Banach $\mathbb{K}$-algebra of bounded continuous functions defined from $\mathbb{E}$ to $\mathbb{K}$. Then $\|f\|_{0} \leq\|f\|_{s p} \leq\|f\| \forall f \in T$. Moreover, given $f \in T$ satisfying $\|f\|_{s p}<1$, then $\lim _{n \rightarrow+\infty}\left\|f^{n}\right\|=0$.

Proof: The norm $\|\cdot\|_{0}$ is power multiplicative and classically it is bounded by the norm $\|$. $\|$ of $T$, it is then bounded by $\|$. $\|_{s p}$. The last claim is immediate.

Definition. Let $S$ be a $\mathbb{K}$-subalgebra of $\mathcal{A}$. We say that $(S,\|\cdot\|)$ is semi-admissible if it is a Banach algebra satisfying the following two properties:

1) For every $O \in \mathbb{B}(\mathbb{E})$, the characteristic function of $O$ belongs to $S$.

2) For every $f \in S$ such that $\inf \{|f(x)| \mid x \in \mathbb{E}\}>0, f$ is invertible in $S$. Moreover the semi-admissible algebra $S$ will be called admissible if $\|f\|_{0}=\|f\|_{s p} \forall f \in S$.

Given a subset $X$ of $S$, we call spectral closure of $X$ denoted by $\widetilde{X}$ the closure of $X$ with respect to the semi-norm $\|.\|_{s p}$ and $X$ will be said to be spectrally closed if $X=\widetilde{X}$. Moreover, $X$ will be said to be uniformly closed if it is closed with respect to the uniform norm and we call uniform closure of $X$ the closure of $X$ with respect to the semi-norm $\|\cdot\|_{0}$.

Throughout the paper the algebra $S$ will be supposed to be semi-admissible.

Let $f \in \mathcal{A}$ be such that $\inf \{|f(x)| \mid x \in \mathbb{E}\}>0$, it is clear that $\frac{1}{f}$ belongs to $\mathcal{A}$. On the other hand, $\mathcal{A}$ is complete with respect to the uniform norm, then we have the following statement: 
Proposition 1.4: The Banach $\mathbb{K}$-algebra $\mathcal{A}$ is admissible.

The following theorem 1.6 shows another example of admissible algebra which is a bit less immediate. In Theorem 1.8, we can see that in various cases, this algebra is strictly included in $\mathcal{A}$.

Lemma 1.5. Let $\left(O_{i}\right)_{i=1, \ldots, n}$ be a finite cover of $\mathbb{E}$ with clopen sets. Then there exists a finite cover $\left(B_{j}\right)_{j=1, \ldots, p}$ of $\mathbb{E}$ where the sets $B_{j}$ are not empty, clopen, pairwise disjoint and such that every $B_{j}$ is contained in some $O_{i}$.

Proof. To the system $\left(O_{i}\right)_{i=1, \ldots, n}$, associate the system $\left(O_{i}^{\prime}\right)_{i=1, \ldots, 2 n}$ where $O_{i}^{\prime}=O_{i}$ if $1 \leq$ $i \leq n$ and $O_{i}^{\prime}=X \backslash O_{i-n}$ otherwise. For every $x \in \mathbb{E}$ define $I_{x}=\left\{i \in\{1, \ldots n\}: x \in O_{i}^{\prime}\right\}$ and consider the following equivalence relation on $\mathbb{E}$ : $x(R) y$ if and only if $I_{x}=I_{y}$. For any $x \in \mathbb{E}$ the equivalence class of $x$ is equal to $\cap_{i \in I_{x}} O_{i}^{\prime}$ and it is clopen since so are the $O_{i}^{\prime}$. Then the equivalence classes $\left(B_{j}\right)_{j=1, \ldots, p}$ satisfy the expected properties.

Theorem 1.6. Let $\mathcal{T}$ be the $\mathbb{K}$-subalgebra of $\mathcal{A}$ generated by the characteristic functions of all clopen sets of $\mathbb{E}$ and let $\mathcal{G}$ be its closure in $\mathcal{A}$ (for the uniform convergence \|\|$_{0}$ on $\mathbb{E})$. Then $\mathcal{G}$ is admissible.

Proof. One just has to prove Property 2) in the definition of a semi-admissible algebra.

First we check that if $g \in \mathcal{T}$ is such that $\inf \{|g(x)|: x \in \mathbb{E}\}=m>0$ then $\frac{1}{g} \in \mathcal{T}$. Since $g \in \mathcal{T}$ there exists a finite cover $\left(O_{i}\right)_{i=1, \ldots, n}$ of $\mathbb{E}$ with clopen sets and scalars $\left(\lambda_{i}\right)_{1 \leq i \leq n}$ in $\mathbb{K}$ such that $g=\sum_{i=1}^{n} \lambda_{i} u_{i}$ where $u_{i}$ is the characteristic function of the clopen $O_{i}$. Using the preceding lemma we get a finite cover $\left(B_{j}\right)_{j=1, \ldots, p}$ of $\mathbb{E}$ where the sets $B_{j}$ are not empty, clopen, pairwise disjoint and such that every $B_{j}$ is contained in some $O_{i}$. Then there exist scalars $\left(\beta_{j}\right)_{1 \leq j \leq p}$ in $\mathbb{K}$ such that $g=\sum_{j=1}^{p} \beta_{j} e_{j}$ where $e_{j}$ is the characteristic function of the clopen $B_{j}$. For every $j$ we get $\left|\beta_{j}\right| \geq m>0$, then it is clear that $\frac{1}{g}=\sum_{j=1}^{p} \frac{1}{\beta_{j}} e_{j}$ and $\frac{1}{g} \in \mathcal{T}$.

Now consider any $f \in \mathcal{G}$ such that $\inf \{|f(x)|: x \in \mathbb{E}\}=m>0$. For every $\epsilon>0$ such that $\epsilon<\frac{1}{m}$ we have $\epsilon m^{2}<m$ and we can consider some $g \in \mathcal{T}$ such that $\|f-g\|_{0} \leq \epsilon m^{2}$. Since $\epsilon m^{2}<m$, we get $|f(x)|=|g(x)|$ for every $x \in \mathbb{E}$ and then $\inf \{|g(x)|: x \in \mathbb{E}\}=m$. Next $\frac{1}{g} \in \mathcal{T}$ and we have for every $x \in \mathbb{E}:\left|\frac{1}{f(x)}-\frac{1}{g(x)}\right|=\frac{|f(x)-g(x)|}{|f(x) g(x)|} \leq \frac{\|f-g\|_{0}}{m^{2}} \leq \epsilon$. This proves that $\frac{1}{f} \in \mathcal{G}$, which ends the proof.

The following proposition is Example 3E, chap.3 in [19].

Proposition 1.7: The algebra $\mathcal{G}$ is the $\mathbb{K}$-algebra of the continuous functions from $\mathbb{E}$ to $\mathbb{K}$ such that the closure of $f(\mathbb{E})$ in $\mathbb{K}$ is compact. In particular when $\mathbb{K}$ is locally compact or $\mathbb{E}$ is compact then $\mathcal{G}=\mathcal{A}$. 
In order to prove Theorem 1.9, we must recall the following classical proposition:

Proposition 1.8 : The field $\mathbb{K}$ is locally compact if and only if its valuation group is discrete and its residue class field is finite. [1], (Proposition 2.3.3) .

Theorem 1.9: Suppose that $\mathbb{E}$ contains a sequence $\left(a_{n}\right)_{n \in \mathbb{N}}$ such that $\inf _{n \neq m}\left(\delta\left(a_{n}, a_{m}\right)\right)>0$ and that $\mathbb{K}$ is not locally compact. Then $\mathcal{G}$ is strictly included in $\mathcal{A}$.

Proof: We put $s=\inf _{n \neq m}\left(\delta\left(a_{n}, a_{m}\right)\right)$. Suppose first that the valuation group of $\mathbb{K}$ is dense. We can consider a partition of $\mathbb{E}$ by an infinite family of balls $d_{\mathbb{E}}\left(b_{i}, s^{-}\right)$.

Suppose first that the valuation group of $\mathbb{K}$ is dense. Then we can define a bounded mapping $\psi$ from $\mathbb{E}$ into $\mathbb{K}$ such that $\psi(x)$ is constant in each ball $d_{\mathbb{E}}\left(b_{i}, s^{-}\right)$, such that $\left|\psi\left(a_{n}\right)-\psi\left(a_{m}\right)\right| \geq 1$ and such that $|\psi(x)| \geq 1 \forall x \in \mathbb{E}$. Particularly, $|\psi(x)|$ takes infinitely many values. Suppose that $\mathcal{T}$ is dense in $\mathcal{A}$. Following the same process as in the proof of Theorem 1.6 above, we can construct a function $g \in \mathcal{T}$ such that $|\psi(x)|=|g(x)|=\lambda_{j} \forall x \in$ $\mathbb{E}$. But $|\psi(x)|$ then only takes finitely many values, a contradiction.

Similarly, suppose now that the residue class field of $\mathbb{K}$ is infinite. Let us consider a sequence of distinct disks $\left(d\left(\mu_{n}, 1^{-}\right)\right)_{n \in \mathbb{N}}$ in the unit circle and and put $B_{n}=d\left(\mu_{n}, 1^{-}\right)$. Now, consider a sequence of balls $d_{\mathbb{E}}\left(a_{n}, s^{-}\right)$in $\mathbb{E}$ and an element $f$ of $\mathcal{A}$ constant in $d_{\mathbb{E}}\left(a_{n}, s\right)$ and such that $f\left(a_{n}\right)$ belongs to $B_{n}$. Suppose that $f$ is in the closure of $\mathcal{T}$. Then there exists $g \in \mathcal{T}$ such that $|f(x)-g(x)|<1$ for any $x \in \mathbb{E}$. In particular, we get that $g\left(a_{n}\right) \in B_{n}$ for every $n$. Thus, $g$ should take infinitely many values, a contradiction.

Definition: Recall that an element $x$ of a normed $\mathbb{K}$-algebra $A$ is called $a$ topological divisor of zero if $\inf _{y \in A,\|y\| \geq 1}\|x y\|=0$.

Theorem 1.10: Suppose that $\mathbb{E}$ has no isolated points. Let $T$ be an admissible Banach $\mathbb{K}$-algebra complete with respect to the norm $\|.\|_{0}$. An element of $T$ is a topological divisor of zero if and only if it is not invertible.

Proof: It is obvious that an invertible element of $T$ is not a topological divisor of zero. Now, consider an element $f \in T$ that is not invertible. Then $\inf _{x \in \mathbb{E}}|f(x)|=0$. Therefore, there exists a sequence of disks $\left(d_{\mathbb{E}}\left(a_{n}, r_{n}\right)\right)_{n \in \mathbb{N}}$ with $\lim _{n \rightarrow \infty} r_{n}=0$, such that $|f(x)| \leq$ $\frac{1}{n}, \forall x \in d_{\mathbb{E}}\left(a_{n}, r_{n}\right), \forall n \in \mathbb{N}^{*}$. For each $n \in \mathbb{N}$, let $h_{n}$ be the characteristic function of $d_{\mathbb{E}}\left(a_{n}, r_{n}\right)$. Then $h_{n}$ belongs to $T$ and satisfies $\left\|h_{n}\right\|_{0}=1 \forall n \in \mathbb{N}^{*}$. On the other hand, we have $\left\|f h_{n}\right\|_{0} \leq \frac{1}{n}$, hence $\lim _{n \rightarrow+\infty} f h_{n}=0$.

More notations and definitions: Let $\mathcal{F}$ be a filter on $\mathbb{E}$. Given a function $f$ from $\mathbb{E}$ to $\mathbb{K}$ admitting a limit along $\mathcal{F}$, we will denote by $\lim _{\mathcal{F}} f(x)$ this limit.

Given a filter $\mathcal{F}$ on $\mathbb{E}$, we will denote by $\mathcal{I}(\mathcal{F}, S)$ the ideal of the $f \in S$ such that $\lim _{\mathcal{F}} f(x)=0$. Notice that the unity does not belong to $\mathcal{I}(\mathcal{F}, S)$, so $\mathcal{I}(\mathcal{F}, S) \neq S$. 
Given $a \in \mathbb{E}$, we will denote by $\mathcal{I}(a, S)$ the ideal of the $f \in S$ such that $f(a)=0$ and by $\mathcal{I}^{\prime}(a, S)$ the ideal of the $f \in S$ such that there exists an open neighborhood $L$ of $a$ such that $f(x)=0 \forall x \in L$.

We will denote by $\operatorname{Max}(S)$ the set of maximal ideals of $S$ and by $\operatorname{Max}_{\mathbb{E}}(S)$ the set of maximal ideals of $S$ of the form $\mathcal{I}(a, S), a \in \mathbb{E}$.

Given a set $F$, we will denote by $U(F)$ the set of ultrafilters on $F$.

Two ultrafilters $\mathcal{U}, \mathcal{V}$ on $\mathbb{E}$ will be said to be sticked if for every closed subsets $H \in$ $\mathcal{U}, G \in \mathcal{V}$, we have $H \cap G \neq \emptyset$.

We will denote by $(\mathcal{R})$ the relation defined on $U(\mathbb{E})$ as $\mathcal{U}(\mathcal{R}) \mathcal{V}$ if $\mathcal{U}$ and $\mathcal{V}$ are sticked $[14]$.

Remark 1: Relation $(\mathcal{R})$ is not the equality between ultrafilters, even when the ultrafilters are not convergent. In [17], Labib Haddad introduced the following equivalence relation $(\mathcal{H})$ on ultrafilters. Given two ultrafilters $\mathcal{U}, \mathcal{V}$ we write $\mathcal{U}(\mathcal{H}) \mathcal{V}$ if there exists an ultrafilter $\mathcal{W}$ such that every closed set $L$ lying in $\mathcal{W}$ also lies in $\mathcal{U}$ and similarly, every closed set $L$ lying in $\mathcal{W}$ also lies in $\mathcal{V}$. So, Relation $(\mathcal{H})$ is clearly thinner than Relation $(\mathcal{R})$. However, it is shown that two ultrafilters $\mathcal{U}, \mathcal{V}$ satisfying $\mathcal{U}(\mathcal{H}) \mathcal{V}$ may be distinct without converging.

The following lemma is classical [7]:

Lemma 1.11: Given $\mathcal{U} \in U(\mathbb{E})$ and a subset $X$ of $\mathbb{E}$, then either $X \in \mathcal{U}$ or $(\mathbb{E} \backslash X) \in \mathcal{U}$.

\section{Theorem 1.12:}

1) if $F$ and $G$ are disjoint closed subsets of $\mathbb{E}$ then there exists a clopen $O$ such that $F \subset O$ and $G \subset(\mathbb{E} \backslash O)$.

This is the case when $\delta(F, G)>0$.

2) If $\mathcal{U}$ and $\mathcal{V}$ are ultrafilters on $\mathbb{E}$ then they are sticked if and only if they contain the same clopen sets.

In particular if $\mathcal{U}, \mathcal{V}$ are not sticked, there exist disjoint clopen subsets $H$ and $L$ of $\mathbb{E}$ such that $H \in \mathcal{U}, H \notin \mathcal{V}$ and $L \in \mathcal{V}, L \notin \mathcal{U}$.

\section{Proof:}

1) For each $x \in F$ take $r_{x}>0$ such that $d\left(x, r_{x}^{-}\right) \cap G=\emptyset$ and define the open set $O=\bigcup_{x \in F} d\left(x, r_{x}^{-}\right)$. We clearly have $F \subset O$ and $G \subset \mathbb{E} \backslash O$. Let us prove that $O$ is closed.

Let $y \in \bar{O}$. For every $n \in \mathbb{N}^{*}$, there exists $x_{n} \in F$ such that $d\left(y, \frac{1}{n}^{-}\right) \cap d\left(x_{n}, r_{x_{n}}^{-}\right) \neq \emptyset$, then let $y_{n} \in d\left(y, \frac{1}{n}^{-}\right) \cap d\left(x_{n}, r_{x_{n}}^{-}\right)$.

First assume that $\inf \left\{r_{x_{n}}: n \in \mathbb{N}^{*}\right\}=m>0$. Take $n \in \mathbb{N}^{*}$ such that $\frac{1}{n}<m$. Since the distance is ultrametric we then have: $d\left(y, \frac{1}{n}^{-}\right)=d\left(y_{n}, \frac{1}{n}^{-}\right) \subset d\left(y_{n}, r_{x_{n}}^{-}\right)=d\left(x_{n}, r_{x_{n}}^{-}\right)$. Finally $y \in O$.

Assume now that $\inf \left\{r_{x_{n}}: n \in \mathbb{N}^{*}\right\}=0$. There exists a subsequence $\left(x_{n_{k}}\right)_{k}$ such that $\left(r_{x_{n_{k}}}\right)_{k}$ tends to 0 . Then we immediately get that $\left(x_{n_{k}}\right)_{k}$ tends to $y$ since $\left(y_{n_{k}}\right)_{k}$ tends to $y$. So $y \in \bar{F}=F$ and again $y \in O$. 
2) If $\mathcal{U}$ and $\mathcal{V}$ are sticked then for every clopen $O \in \mathcal{U}$ we necessarily have $O \in \mathcal{V}$. Otherwise using the preceding lemma the clopen $\mathbb{E} \backslash O$ is in $\mathcal{V}$ so $\mathcal{U}$ and $\mathcal{V}$ cannot be sticked. Conversely, if $\mathcal{U}$ and $\mathcal{V}$ contain the same clopen sets then using the preceding property 1), for every closed sets $F \in \mathcal{U}$ and $G \in \mathcal{V}$ we necessarily get $F \cap G \neq \emptyset$, otherwise taking a clopen $O$ such in 1) we have $O \in \mathcal{U}$ and $O \notin \mathcal{V}$ since $\mathbb{E} \backslash O \in \mathcal{V}$.

In particular if $\mathcal{U}$ and $\mathcal{V}$ are not sticked then taking some clopen $H$ in $\mathcal{U}$ which is not in $\mathcal{V}$, we have $(\mathbb{E} \backslash H) \in \mathcal{V}$ and putting $L=\mathbb{E} \backslash H, H$ and $L$ are clopen sets satisfying the expected property.

Corollary 1.12.a: Let $\mathcal{U}, \mathcal{V}$ be two ultrafilters on $E$ that are not sticked. There exist clopen subsets $H \in \mathcal{U}, L \in \mathcal{V}$ and $f \in S$ such that $f(x)=1 \forall x \in H, f(x)=0 \forall x \in L$.

Lemma 1.13 is classical:

Lemma 1.13: Let $\mathcal{U}$ be an ultrafilter on $\mathbb{E}$. Let $f$ be a bounded function from $\mathbb{E}$ to $\mathbb{I K}$. The function $|f|$ from $\mathbb{E}$ to $\mathbb{R}_{+}$defined as $|f|(x)=|f(x)|$ admits a limit along $\mathcal{U}$. Moreover, if $\mathbb{K}$ is locally compact, then $f(x)$ admits a limit along $\mathcal{U}$.

Recall that for any normed $\mathbb{K}$-algebra $(G,\|\cdot\|)$, the closure of an ideal of $G$ is an ideal of $G$. Lemmas 1.14 and 1.15 are immediate:

Lemma 1.14: The spectral closure of an ideal of $S$ is an ideal of $S$.

Lemma 1.15: Let $X \subset S$ be spectrally closed. Then $X$ is closed with respect to the norm of $S$. Let $Y \subset S$ be uniformly closed. Then it is spectrally closed.

Now we can recall a classical result known in ultrametric analysis as in Archimedean analysis.

Proposition 1.16: Every maximal ideal $\mathcal{M}$ of $S$ is spectrally closed.

Proof: By Lemma 1.14 the spectral closure $\widetilde{\mathcal{M}}$ of $\mathcal{M}$ is an ideal. If $\mathcal{M}$ is not spectrally closed, then $\widetilde{\mathcal{M}}=S$, hence there exists $t \in S$ such that $1-t \in \mathcal{M}$ and $\|t\|_{s p}<1$. Consequently, by Proposition $1.3 \lim _{n \rightarrow+\infty}\left\|t^{n}\right\|=0$, therefore the series $\left(\sum_{n=0}^{\infty} t^{n}\right)$ converges and $\left(\sum_{n=0}^{\infty} t^{n}\right)(1-t)=1$ and hence the unity belongs to $\mathcal{M}$, a contradiction.

Proposition 1.17 now is easy:

Proposition 1.17: Given an ultrafilter $\mathcal{U}$ on $\mathbb{E}, \mathcal{I}(\mathcal{U}, S)$ is a prime ideal. Moreover, $\mathcal{I}(\mathcal{U}, S)$ is uniformly closed and hence is spectrally closed and closed for the topology of $S$.

Proof: Since $\mathcal{U}$ is an ultrafilter, it is obvious that $\mathcal{I}(\mathcal{U}, S)$ is prime. Indeed, given $f \in S$, by Lemma 1.13, $|f(x)|$ admits a limit along $\mathcal{U}$ and hence, if $f, g \in S$ are such that 
$\lim _{\mathcal{U}} f(x) g(x)=0$, then either $\lim _{\mathcal{U}} f(x)=0$ or $\lim _{\mathcal{U}} g(x)=0$, hence either $f$ or $g$ belongs to $\mathcal{I}(\mathcal{U}, S)$.

Let us now check that $\mathcal{I}(\mathcal{U}, S)$ is uniformly closed. Indeed let $g$ in the closure of $\mathcal{I}(\mathcal{U}, S)$ with respect to $\|$. $\|_{0}$, let $b=\lim _{\mathcal{U}}|g(x)|$ and suppose $b>0$. There exists $f \in \mathcal{I}(\mathcal{U}, S)$ such that $\|f-g\|_{0}<b$ and then

$$
b=\left.\left|\lim _{\mathcal{U}}\right| f(x)\left|-\lim _{\mathcal{U}}\right| g(x)\right|_{\infty} \leq \lim _{\mathcal{U}}|f(x)-g(x)| \leq\|f-g\|_{0}<b,
$$

a contradiction showing that $\mathcal{I}(\mathcal{U}, S)$ is uniformly closed. Therefore, it is spectrally closed and closed for the topology of $S$.

The following Theorem 1.18 is proved in [14].

Theorem 1.18: Let $\mathcal{U}, \mathcal{V}$ be two ultrafilters on $\mathbb{E}$. Then $\mathcal{I}(\mathcal{U}, S)=\mathcal{I}(\mathcal{V}, S)$ if and only if $\mathcal{U}$ and $\mathcal{V}$ are sticked.

Proof: First, if $\mathcal{U}$ and $\mathcal{V}$ are not sticked, by Corollary 1.12.a we have $\mathcal{I}(\mathcal{U}, S) \neq \mathcal{I}(\mathcal{V}, S)$. Now, suppose that $\mathcal{U}, \mathcal{V}$ are sticked. By Theorem 1.12, then they contain the same clopen sets. But for every $f \in S$ and $\epsilon>0$ the set $L_{\epsilon}=\{x \in \mathbb{E}:|f(x)| \leq \epsilon\}$ is clopen and we have: $f \in \mathcal{I}(\mathcal{U}, S) \Longleftrightarrow \forall \epsilon>0, L_{\epsilon} \in \mathcal{U}$ and hence $L_{\epsilon} \in \mathcal{V}$. Consequently, $\forall \epsilon>0, L_{\epsilon} \in \mathcal{V}$ and hence $f$ belongs to $\mathcal{I}(\mathcal{V}, S)$. Thus $\mathcal{I}(\mathcal{U}, S) \subset \mathcal{I}(\mathcal{V}, S)$ and similarly, $\mathcal{I}(\mathcal{V}, S) \subset \mathcal{I}(\mathcal{U}, S)$, therefore $\mathcal{I}(\mathcal{V}, S)=\mathcal{I}(\mathcal{U}, S)$

Corollary 1.18.a: Relation $(\mathcal{R})$ is an equivalence relation on $U(\mathbb{E})$.

Theorem 1.19 looks like certain Bezout-Corona statements [18]:

Theorem 1.19: Let $f_{1}, \ldots, f_{q} \in S$ satisfy $\inf _{x \in \mathbb{E}}\left(\max _{1 \leq j \leq q}\left|f_{j}(x)\right|\right)>0$. Then there exist $g_{1}, \ldots, g_{q} \in S$ such that $\sum_{j=1}^{q} f_{j}(x) g_{j}(x)=1 \forall x \in \mathbb{E}$.

Proof: $\quad$ Let $M=\inf _{x \in \mathbb{E}}\left(\max _{1 \leq j \leq q}\left|f_{j}(x)\right|\right)$. Let $E_{j}=\left\{x \in \mathbb{E}|| f_{j}(x) \mid \geq M\right\}, j=1, \ldots, q$ and let $F_{j}=\bigcup_{m=1}^{j} E_{m}, j=1, \ldots, q$. Let $g_{1}(x)=\frac{1}{f_{1}(x)} \forall x \in E_{1}$ and $g_{1}(x)=0 \forall x \in \mathbb{E} \backslash E_{1}$. Since $\left|f_{1}(x)\right| \geq M \forall x \in E_{1},\left|g_{1}(x)\right|$ is clearly bounded. By Lemma 1.2 each $E_{j}$ is obviously clopen and so is each $F_{j}$. And since $f_{1}$ is continuous $g_{1}$ is continuous, hence belongs to $S$.

Suppose now we have constructed $g_{1}, \ldots, g_{k} \in S$ satisfying $\sum_{j=1}^{k} f_{j} g_{j}(x)=1 \forall x \in F_{k}$ and $\sum_{j=1}^{k} f_{j} g_{j}(x)=0 \forall x \in \mathbb{E} \backslash F_{k}$. Let $g_{k+1}$ be defined on $\mathbb{E}$ by $g_{k+1}(x)=\frac{1}{f_{k+1}(x)} \forall x \in$ $F_{k+1} \backslash F_{k}$ and $g_{k+1}(x)=0 \forall x \in \mathbb{E} \backslash\left(F_{k+1} \backslash F_{k}\right)$. Since $F_{k}$ and $F_{k+1}$ are clopens, so is $\mathbb{E} \backslash\left(F_{k+1} \backslash F_{k}\right)$ and consequently, $g_{k+1}$ is continuous. Similarly as for $g_{1}$, since 
$\left|f_{k+1}(x)\right| \geq M \forall x \in E_{k+1},\left|g_{k+1}(x)\right|$ is clearly bounded, hence $g_{k+1}$ belongs to $S$. Now we can check that $\sum_{j=1}^{k+1} f_{j} g_{j}(x)=1 \forall x \in F_{k+1}$ and $\sum_{j=1}^{k} f_{j} g_{j}(x)=0 \forall x \in \mathbb{E} \backslash F_{k+1}$. So, by a finite induction, we get functions $g_{1}, \ldots, g_{q} \in S$ such that $\sum_{j=1}^{q} f_{j} g_{j}(x)=1 \forall x \in \mathbb{E}$, which ends the proof.

Notation: Let $f \in S$ and let $\epsilon>0$. We set $D(f, \epsilon)=\{x \in \mathbb{E}|| f(x) \mid \leq \epsilon\}$.

Corollary 1.19.a: Let $I$ be an ideal of $S$ different from $S$. The family of sets $\{D(f, \epsilon), f \in I, \epsilon>0\}$ generates a filter $\mathcal{F}_{I, S}$ on $\mathbb{E}$ such that $I \subset \mathcal{I}\left(\mathcal{F}_{I, S}, S\right)$.

\section{Maximal and prime ideals of $S$}

Except Theorem 2.4 and its corollaries, most of the results of this paragraph were given in [14] for the algebra $\mathcal{A}$.

Theorem 2.1: $\quad$ Let $\mathcal{M}$ be a maximal ideal of $S$. There exists an ultrafilter $\mathcal{U}$ on $\mathbb{E}$ such that $\mathcal{M}=\mathcal{I}(\mathcal{U}, S)$. Moreover, $\mathcal{M}$ is of codimension 1 if and only if every element of $S$ converges along $\mathcal{U}$. In particular if $\mathcal{U}$ is convergent, then $\mathcal{M}$ is of codimension 1.

Proof: Indeed, by Corollary 1.19.a, we can consider the filter $\mathcal{F}_{\mathcal{M}, S}$ and we have $\mathcal{M} \subset$ $\mathcal{I}\left(\mathcal{F}_{\mathcal{M}, S}, S\right)$. Let $\mathcal{U}$ be an ultrafilter thinner than $\mathcal{F}_{\mathcal{M}, S}$. So, we have $\mathcal{M} \subset \mathcal{I}\left(\mathcal{F}_{\mathcal{M}, S}, S\right) \subset$ $\mathcal{I}(\mathcal{U}, S)$. But since $\mathcal{M}$ is a maximal ideal, either $\mathcal{M}=\mathcal{I}(\mathcal{U}, S)$, or $\mathcal{I}(\mathcal{U}, S)=S$. But obviously, $\mathcal{I}(\mathcal{U}, S) \neq S$, hence $\mathcal{M}=\mathcal{I}(\mathcal{U}, S)$.

Now assume that $\mathcal{M}$ is of codimension 1 and let $\chi$ be the $\mathbb{K}$-algebra homomorphism from $S$ to $\mathbb{K}$ admitting $\mathcal{M}$ for kernel. Let $f \in S$ and let $b=\chi(f)$. Then $f-b$ belongs to the kernel of $\mathcal{M}$, hence $\lim _{\mathcal{U}} f(x)-b=0$ that is $\lim _{\mathcal{U}} f(x)=b$ therefore every element of $S$ converges along $\mathcal{U}$.

Conversely if every element of $S$ admits a limit along $\mathcal{U}$ then the mapping $\chi$ which associates to each $f \in S$ its limit along $\mathcal{U}$ is a $\mathbb{K}$-algebra homomorphism from $S$ to $\mathbb{K}$ admitting $\mathcal{M}$ for kernel, therefore $\mathcal{M}$ is of codimension 1 .

In particular if $\mathcal{U}$ converges to a point $a$, then each $f$ in $S$ converges to $f(a)$ along $\mathcal{U}$.

By Lemma 1.13 and Theorem 2.1, the following corollary is immediate:

Corollary 2.1.a: Let $\mathbb{K}$ be a locally compact field. Then every maximal ideal of $S$ is of codimension 1.

Remark 3: If $\mathbb{I K}$ is locally compact, a maximal ideal of codimension 1 of $S$ is not necessarily of the form $\mathcal{I}(\mathcal{U}, S)$ where $\mathcal{U}$ is a Cauchy ultrafilter. Suppose that $\mathbb{E}$ admits a sequence $\left(a_{n}\right)_{n \in \mathbb{N}}$ such that either it satisfies $\left|a_{n}-a_{m}\right|=r \forall n \neq m$, or the sequence $\left|a_{n+1}-a_{n}\right|$ is strictly increasing. Let $\mathcal{U}$ be an ultrafilter thinner than the sequence $\left(a_{n}\right)_{n \in \mathbb{N}}$. 
Consider now a function $f \in S$. Since $\mathbb{K}$ is locally compact, $f(x)$ does converge along $\mathcal{U}$ to a point $b \in \mathbb{K}$. In that way, we can define a homomorphism $\chi$ from $S$ onto $\mathbb{K}$ as $\chi(g)=\lim _{\mathcal{U}} g(x)$ and therefore $\mathbb{K}$ is the quotient $\frac{S}{\operatorname{Ker}(\chi)}$. So $\mathcal{I}(\mathcal{U}, S)$ is a maximal ideal of codimension 1.

Notation: Following notations of [14], we will denote by $Y_{(\mathcal{R})}(\mathbb{E})$ the set of equivalence classes on $U(\mathbb{E})$ with respect to Relation $(\mathcal{R})$.

By Theorem 1.18, we can get Corollary 2.1.b:

Corollary 2.1.b: Let $\mathcal{M}$ be a maximal ideal of $S$. There exists a unique $\mathcal{H} \in Y_{(\mathcal{R})}(\mathbb{E})$ such that $\mathcal{M}=\mathcal{I}(\mathcal{U}, S)$ for every $\mathcal{U} \in \mathcal{H}$.

Conversely, Theorem 2.2 now characterizes all maximal ideals of $S$.

Theorem 2.2: $\quad$ Let $\mathcal{U}$ be an ultrafilter on $\mathbb{E}$. Then $\mathcal{I}(\mathcal{U}, S)$ is a maximal ideal of $S$.

Proof: Let $I=\mathcal{I}(\mathcal{U}, S)$ and let $\mathcal{M}$ be a maximal ideal of $S$ containing $I$. Then by Theorem 2.1 there exists an ultrafilter $\mathcal{V}$ such that $\mathcal{M}=\mathcal{I}(\mathcal{V}, S)$. Suppose now $\mathcal{I}(\mathcal{U}, S) \neq$ $\mathcal{I}(\mathcal{V}, S)$. Then, $\mathcal{U}$ and $\mathcal{V}$ are not sticked. Consequently, by Theorem 1.12 there exists a clopen subset $F \in \mathcal{V}$ that does not belong to $\mathcal{U}$ and hence its characteristic function $u \in S$ belongs to $\mathcal{I}(\mathcal{U}, S)$ but does not belong to $\mathcal{I}(\mathcal{V}, S)$. Thus, $u$ belongs to $I$ but does not belong to $\mathcal{M}$, a contradiction to the hypothesis.

By Corollary 2.1.b and Theorem 2.2, we can derive the following Corollary 2.2.a:

Corollary 2.2.a: The mapping that associates to each maximal ideal $\mathcal{M}$ of $S$ the class with respect to $(\mathcal{R})$ of ultrafilters $\mathcal{U}$, such that $\mathcal{M}=\mathcal{I}(\mathcal{U}, S)$, is a bijection from $\operatorname{Max}(S)$ onto $Y_{(\mathcal{R})}(\mathbb{E})$.

Remark 4: Let $\mathcal{F}$ be a Cauchy filter on $\mathbb{E}$ admitting a limit limit $a \in \mathbb{E}$ and let $\mathcal{M}=\mathcal{I}(\mathcal{F}, S)$. Then every function $f \in S$ converges to a limit $\theta(f)$ along $\mathcal{F}$ and $\mathcal{M}$ is a maximal ideal of codimension 1. Indeed, let $f \in S$. Since $f$ is continuous, then $f(x)$ converges to a point $\theta(f)=f(a)$ in $\mathbb{K}$. Consider now the mapping $\theta$ from $S$ into $\mathbb{K}$ : it is an algebra morphism whose kernel is $\mathcal{M}$ and whose image is $\mathbb{K}$. Consequently, $\frac{S}{\mathcal{M}}=\mathbb{K}$, therefore $\mathcal{M}$ is a maximal ideal of codimension 1 .

Notation: For any subset $F$ of $\mathbb{E}$, we denote by $u_{F}$ its characteristic function. Let $\mathcal{M}$ be a maximal ideal of $S$ and let $\mathcal{U} \in U(\mathbb{E})$ be such that $\mathcal{M}=\mathcal{I}(\mathcal{U}, S)$. By Theorems 1.18 we can define the set $\mathcal{O}_{\mathcal{M}}$ of all clopen subsets of $\mathbb{E}$ which belong to $\mathcal{U}$. We then denote by $\mathcal{C}_{\mathcal{M}}$ the set $\left\{u_{\mathbb{E} \backslash L} \mid L \in \mathcal{O}_{\mathcal{M}}\right\}$ and by $\mathcal{J}_{\mathcal{M}}$ the set of all functions $f \in S$ which are equal to 0 on some $L \in \mathcal{O}_{\mathcal{M}}$.

Given $a \in \mathbb{E}$, we will denote by $\mathcal{I}^{\prime}(a, S)$ the ideal of the functions $f \in S$ equal to 0 on an open subset of $\mathbb{E}$ containing $a$.

Theorem 2.3: $\quad$ Let $\mathcal{M}$ be a maximal ideal of $S$. 
1) $\mathcal{J}_{\mathcal{M}}$ is an ideal of $S$ containing $\mathcal{C}_{\mathcal{M}}$,

2) $\mathcal{J}_{\mathcal{M}}$ is the ideal of $S$ generated by $\mathcal{C}_{\mathcal{M}}$ and $\mathcal{J}_{\mathcal{M}}=\left\{f u \mid f \in S, u \in \mathcal{C}_{\mathcal{M}}\right\}$,

3) If $\mathcal{P}$ is a prime ideal of $S$ contained in $\mathcal{M}$, then $\mathcal{J}_{\mathcal{M}} \subset \mathcal{P}$.

4) if $\mathcal{M}=\mathcal{I}(a, S)$, then $\mathcal{I}^{\prime}(a, S)=\mathcal{J}_{\mathcal{M}}$.

Proof: 1) Let us check that $\mathcal{J}_{\mathcal{M}}$ is an ideal of $S$. Let $f, g \in \mathcal{J}_{\mathcal{M}}$. So, there exist $F, G \in \mathcal{O}_{\mathcal{M}}$ such that $f(x)=0 \forall x \in F, g(x)=0 \forall x \in G$, hence $f(x)-g(x)=0 \forall x \in F \cap G$. Since $F \cap G$ belongs to $\mathcal{O}_{\mathcal{M}}, f-g$ lies in $\mathcal{J}_{\mathcal{M}}$. And obviously, for every $h \in S$, we have $h(x) f(x)=0 \forall x \in F$, hence $f h$ lies in $\mathcal{J}_{\mathcal{M}}$.

Next, $\mathcal{J}_{\mathcal{M}}$ contains $\mathcal{C}_{\mathcal{M}}$ because given $L \in \mathcal{O}_{\mathcal{M}}$, the set $\mathbb{E} \backslash L$ is clopen, then $u_{\mathbb{E} \backslash L}$ belongs to $S$ and is equal to 0 on $L$.

2) Notice that if $f \in S$ and $u \in \mathcal{C}_{\mathcal{M}}$, then by 1) $f u$ belongs to $\mathcal{J}_{\mathcal{M}}$. Conversely, if $f \in \mathcal{J}_{\mathcal{M}}$ and $L \in \mathcal{O}_{\mathcal{M}}$ are such that $f(x)$ is equal to 0 on $L$, then $u_{\mathbb{E} \backslash L}$ belongs to $\mathcal{C}_{\mathcal{M}}$ and $f=f u_{\mathbb{E} \backslash L}$. This proves that $\mathcal{J}_{\mathcal{M}}=\left\{f u \mid f \in S, u \in \mathcal{C}_{\mathcal{M}}\right\}$ and that $\mathcal{J}_{\mathcal{M}}$ is the ideal generated by $\mathcal{C}_{\mathcal{M}}$.

3) It is sufficient to prove that $\mathcal{C}_{\mathcal{M}}$ is included in $\mathcal{P}$. Indeed, let $\mathcal{U} \in U(\mathbb{E})$ be such that $\mathcal{M}=\mathcal{I}(\mathcal{U}, S)$ and let $L \in \mathcal{O}_{\mathcal{M}}$. Then $L \in \mathcal{U}$ and $u_{L} \notin \mathcal{M}$. So, $u_{L} \notin \mathcal{P}$. But $u_{L} . u_{\mathbb{E} \backslash L}=0$. Thus $u_{\mathbb{E} \backslash L}$ belongs to $\mathcal{P}$ since $\mathcal{P}$ is prime.

4) Just notice that $\mathcal{J}_{\mathcal{M}}$ is the set of all functions in $S$ which are equal to 0 on some clopen containing $a$ and that each open neighborhood of $a$ contains a disk $d_{\mathbb{E}}\left(a, r^{-}\right)$, which is clopen.

Corollary 2.3.a: Let $\mathcal{U}$ be an ultrafilter on $\mathbb{E}$ and let $\mathcal{P}$ be a prime ideal included in $\mathcal{I}(\mathcal{U}, S)$. Let $L \in \mathcal{U}$ be clopen and let $H=\mathbb{E} \backslash L$. Then the characteristic function $u$ of $H$ belongs to $\mathcal{P}$.

Theorem 2.4: Let $\mathcal{M}$ be a maximal ideal of $S$. The uniform closure of $\mathcal{J}_{\mathcal{M}}$ is equal to $\mathcal{M}$.

Proof: Let $f \in \mathcal{M}=\mathcal{I}(\mathcal{U}, S)$. Then for every $\epsilon>0$ the set $L=D(f, \epsilon)$ belongs to $\mathcal{U}$ and $L$ is clopen. Therefore $L$ belongs to $\mathcal{O}_{\mathcal{M}}$ and the characteristic function $u$ of $\mathbb{E} \backslash L$ lies in $\mathcal{C}_{\mathcal{M}}$, so that $f u \in \mathcal{J}_{\mathcal{M}}$. But $f(x)-u f(x)=0 \forall x \notin L$ and $|f(x)-u f(x)|=|f(x)| \leq \epsilon \forall x \in L$, so $\|f-u f\|_{0} \leq \epsilon$. Hence $\mathcal{M}$ is the uniform closure of $\mathcal{J}_{\mathcal{M}}$ since, by Proposition 1.16, $\mathcal{M}$ is uniformly closed.

Corollary 2.4.a: Let $\mathcal{P}$ be a prime ideal contained in a maximal ideal $\mathcal{M}$. Then $\mathcal{M}$ is the uniform closure of $\mathcal{P}$.

Corollary 2.4.b: The uniform closure of a prime ideal of $S$ is a maximal ideal of $S$ and a prime ideal of $S$ is contained in a unique maximal ideal of $S$.

Corollary 2.4.c: A prime ideal of $S$ is a maximal ideal if and only if it is uniformly closed.

Using property 4 of theorem 2.3 we get Corollary 2.4.d: 
Corollary 2.4.d: The uniform closure of $\mathcal{I}^{\prime}(a, S)$ is $\mathcal{I}(a, S)$.

Corollary 2.4.e: $\quad$ Let $\mathcal{M}$ be a maximal ideal of $S$. If $S$ is admissible then:

1) $\mathcal{M}$ is the spectral closure of $\mathcal{J}_{\mathcal{M}}$ and the spectral closure of any prime ideal contained in $\mathcal{M}$;

2) a prime ideal is maximal if and only if it is spectrally closed.

\section{Maximal ideals of finite codimension}

The main results of this paragraph were allready obtained in [5]. We recall them with all proofs in order to make easy the conclusions of this article.

Notation: Let $\mathbb{L}$ be a finite algebraic extension of $\mathbb{K}$ provided with the absolute value which extends that of $\mathbb{K}$ and let $t=[\mathbb{L}: \mathbb{K}]$. Let $\mathcal{A}^{e}$ be equal to the $\mathbb{L}$-algebra of the bounded continuous functions of $\mathbb{E}$ into $\mathbb{L}$ and $\widehat{\mathcal{A}}=\mathbb{L} \otimes_{\mathbb{K}} \mathcal{A}$. Since $\mathbb{L}$ is of finite dimension over $\mathbb{I K}$, one obtains an immediate identification of $\mathcal{A}^{e}$ with $\widehat{\mathcal{A}}$

The following Theorem 3.2 holds on all complete valued fields and is proven in [6] (Lemma 7.2). First we must state Lemma 3.1.

Lemma 3.1: $\quad$ Let $\mathbb{L}$ be of the form $\mathbb{L}=\mathbb{K}[a]$. Let $f \in \mathcal{A}^{e}$. Then $f$ is of the form $\sum_{j=0}^{t-1} a^{j} f_{j}, j=0, \ldots, t-1$, with $f_{j} \in \mathcal{A}$.

Theorem 3.2: $\quad$ Let $T=\mathcal{A}$. Suppose there exists a morphism of $\mathbb{K}$-algebra, $\chi$ from $T$ onto $\mathbb{L}$. Then $\chi$ has continuation to a surjective morphism of $\mathbb{L}$-algebra $\widehat{\chi}$ from $\widehat{T}$ to $\mathbb{L}$.

Proof: Suppose first that $\mathbb{L}$ is of the form $\mathbb{K}[a]$. Let $f, g \in \widehat{T}$. Then by Lemma 3.1, $f$ is of the form $\sum_{j=0}^{t-1} a^{j} f_{j}, j=0, \ldots, t-1$ and $g$ is of the form $\sum_{j=0}^{t-1} a^{j} g_{j}, j=0, \ldots, t-1$, where the $f_{j}$ and the $g_{j}$ are functions from $\mathbb{E}$ to $\mathbb{K}$.

We can now define $\widehat{\chi}$ on $\widehat{T}$ as $\widehat{\chi}(f)=\sum_{j=0}^{t-1} a^{j} \chi\left(f_{j}\right)$. Then obviously, $\widehat{\chi}$ is $\mathbb{K}$-linear. On the other hand, for each $q \in \mathbb{N}, a^{q}$ is of the form $P_{q}(a)$ where $P_{q} \in \mathbb{K}[x], \operatorname{deg}\left(P_{q}\right) \leq t-1$. Then $\widehat{\chi}\left(a^{q}\right)=\widehat{\chi}\left(P_{q}(a)\right)=P_{q}(\widehat{\chi}(a))=P_{q}(a)=a^{q}$. Next,

$$
\begin{gathered}
\hat{\chi}(f g)=\widehat{\chi}\left(\left(\sum_{j=0}^{t-1} a^{j} f_{j}\right)\left(\sum_{j=0}^{t-1} a^{j} g_{j}\right)\right)=\widehat{\chi}\left(\sum_{\substack{0 \leq m \leq t-1 \\
0 \leq n \leq t-1}} a^{m+n} f_{m} g_{n}\right) \\
=\sum_{\substack{0 \leq m \leq t-1 \\
0 \leq n \leq t-1}} a^{m+n} \chi\left(f_{m}\right) \chi\left(g_{n}\right)=\left(\sum_{j=0}^{t-1} a^{j} \chi\left(f_{j}\right)\right)\left(\sum_{j=0}^{t-1} a^{j} \chi\left(g_{j}\right)\right)=\chi(f) \chi(g) .
\end{gathered}
$$


Thus, the extension of $\chi$ is proved whenever $\mathbb{L}$ is of the form $\mathbb{K}[a]$. It is then immediate to check that $\widehat{\chi}$ is surjective: since $\widehat{T}$ is a $\mathbb{L}$-algebra, it contains the field $\mathbb{L}$ and every morphism $\widehat{\chi}$ from $\widehat{T}$ obviously satisfies $\widehat{\chi}(c)=c \forall c \in \mathbb{L}$.

Consider now the general case. We can obviously write $\mathbb{L}$ in the form $\mathbb{K}\left[b_{1}, \ldots, b_{q}\right]$. Writing $\mathbb{L}_{j}$ for the extension $\mathbb{K}\left[b_{1}, \ldots, b_{j}\right]$ we have $\mathbb{L}_{j}=\mathbb{L}_{j-1}\left[b_{j}\right]$.

By induction on $j$, using the preceding just proved result, we get that for each $j=$ $1, \ldots, q, \chi$ has continuation to a surjective morphism of $\mathbb{L}_{j}$-algebra, $\widehat{\chi}_{j}$, from $\mathbb{L}_{j} \otimes T$ onto $\mathbb{L}_{j}$. Taking $j=q$ ends the proof.

We are now able to prove that maximal ideals of finite codimension of $S$ are of codimension 1 in two cases: when $S=\mathcal{A}$ and when the field $\mathbb{K}$ is perfect.

Theorem 3.3: Every maximal ideal $\mathcal{M}$ of finite codimension of $\mathcal{A}$ is of codimension 1.

Proof: Let $S=\mathcal{A}$, let $\mathbb{L}$ be the field $\frac{S}{\mathcal{M}}$ and let $S^{\prime}$ be the $\mathbb{L}$-algebra of bounded continuous functions from $\mathbb{E}$ to $\mathbb{L}$. Then $S^{\prime}$ is semi-admissible. Now, let $\chi$ be the $\mathbb{K}$ algebra morphism from $S$ onto $\mathbb{L}$ whose kernel is $\mathcal{M}$. Let $g \in S$ and let $b=\chi(g) \in \mathbb{L}$. By Theorem $3.2 \chi$ admits an extension to a morphism $\widehat{\chi}$ from $S^{\prime}$ to $\mathbb{L}$. Now, since $S^{\prime}$ is semi-admissible and since the kernel of $\widehat{\chi}$ is a maximal ideal $\widehat{\mathcal{M}}$ of $S^{\prime}$, there exists an ultrafilter $\mathcal{U}$ on $\mathbb{E}$ such that $\widehat{\mathcal{M}}=\mathcal{I}\left(\mathcal{U}, S^{\prime}\right)$. Then we have $\widehat{\chi}(g-b)=0$, hence $g-b$ belongs to $\widehat{\mathcal{M}}$, therefore $\lim _{\mathcal{U}} g(x)-b=0$ i.e. $\lim _{\mathcal{U}} g(x)=b$. But since $g \in S, g(x)$ belongs to $\mathbb{K}$ for all $x \in \mathbb{E}$. Therefore, since $\mathbb{K}$ is complete, $b$ belongs to $\mathbb{K}$. But by definition $\chi$ is a surjection from $S$ onto $\mathbb{L}$, hence every value $b$ of $\mathbb{L}$ is the image of some $f \in S$ and hence it lies in $\mathbb{K}$, therefore $\mathbb{L}=\mathbb{K}$.

Theorem 3.3 can be generalized to all semi-admissible algebras provided $\mathbb{K}$ is a perfect field $[5]$.

Henceforth, for the rest of this Paragraph 3, the field $\mathbb{I K}$ is supposed to be perfect.

Proposition 3.4: $\quad$ Let $\mathbb{L}=\mathbb{K}[a]$ be a finite extension of $\mathbb{K}$ of degree $t$, provided with the unique absolute value extending that of $\mathbb{K}$ and let $a_{2}, \ldots, a_{t}$ be the conjugates of a over $\mathbb{K}$, with $a_{1}=a$. Let $\widehat{S}=\mathbb{L} \otimes_{\mathbb{K}} S$ and let $g=\sum_{j=0}^{t-1} a^{j} f_{j}, f_{j} \in S$ be such that $\inf _{\mathbb{E}}|g(x)|>0$. For every $k=1, \ldots, t$, let $g_{k}=\sum_{j=0}^{t-1} a_{k}^{j} f_{j}, f_{j} \in S$. Then $\prod_{k=1}^{t} g_{k}$ belongs to $S$ and $\prod_{k=2}^{t} g_{k}$ belongs to $\widehat{S}$.

Proof: Since $\mathbb{L}=\mathbb{K}\left[a_{1}\right]$ and since $\mathbb{K}$ is perfect, the extension $N=\mathbb{K}\left[a_{1}, \ldots \ldots .,, a_{t}\right]$ is of the form

$$
N=\mathbb{K}\left[a_{1}\right]\left[a_{2}, \ldots \ldots ., a_{t}\right]=\mathbb{L}\left[a_{2}, \ldots \ldots \ldots, a_{t}\right]
$$

and $N$ is a normal extension of $\mathbb{K}$ and of $\mathbb{L}$ respectively.

Thus, assuming that $a_{1}, \ldots \ldots \ldots, a_{s}$ belong to $\mathbb{L}$, we have $\mathbb{L}=\mathbb{K}\left[a_{1}, \ldots \ldots ., a_{s}\right]$ and $N=\mathbb{K}\left[a_{1} \ldots . ., a_{s}, a_{s+1}, \ldots \ldots, a_{t}\right]=\mathbb{K}\left[a_{1}, \ldots \ldots ., a_{s}\right]\left[a_{s+1}, \ldots \ldots ., a_{t}\right]=\mathbb{L}\left[a_{s+1}, \ldots \ldots, a_{t}\right]$. 
Let $G$ be the Gallois group of $N$ over $\mathbb{K}$ and put $G^{\prime}=\{\sigma \in G: \sigma(x)=x, \forall x \in \mathbb{L}\}$ where the extension $N$ over $\mathbb{L}$ is Galoisian, whose Galois group $G(N \mid \mathbb{L})=$ is $G^{\prime}$. The subfield $\mathbb{L}$ of $N$ then corresponds to the subgroup $G^{\prime}$ of $G$ through the Galois correspondance.

Now, given $\sigma \in G$, set $\sigma(g)=\sum_{j=0}^{t-1}(\sigma(a))^{j} f_{j}$. Let $F=\prod_{k=1}^{t} g_{k}=\prod_{\sigma \in G} \sigma(g)$. Then $F$ belongs to $S$ if and only if for every $\tau \in G, \tau(F)=F$. Now, we have

$$
\tau(F)=\prod_{\sigma \in G} \tau \circ \sigma(g)=\prod_{\zeta \in G} \zeta(g)=F,
$$

therefore $F$ belongs to $S$.

On the other hand the roots $a_{i}$, for $s+1 \leq i \leq t$, are conjugate over $\mathbb{L}$. Therefore if $s+1 \leq i \leq t$, there exists $\theta \in G^{\prime}$ such that $a_{i}=\theta\left(a_{s+1}\right)$. It follows that $g_{i}=\sum_{j=0}^{t-1} a_{i}^{j} f_{j}=$ $\sum_{j=0}^{t-1} \theta\left(a_{s+1}\right)^{j} f_{j}=\theta\left(\sum_{j=0}^{t-1} a_{s+1}^{j} f_{j}\right)=\theta\left(g_{s+1}\right)$

Let $H=\prod_{i=s+1}^{t} g_{i}=\prod_{\theta \in G^{\prime}} \theta\left(g_{s+1}\right)$. Then $H$ belongs to $\widehat{S}$ if and only if $\tau(H)=H \forall \tau \in$ $G^{\prime}$. Now, we have $\tau(H)=\prod_{\theta \in G^{\prime}} \tau \circ \theta\left(g_{s+1}\right)=\prod_{\zeta \in G^{\prime}} \zeta\left(g_{s+1}\right)=H$, therefore $H$ belongs to $\widehat{S}$. Consequently, since $\prod_{i=2}^{s} g_{i}$ belongs to $\widehat{S}$, one gets that $\left(\prod_{i=2}^{s} g_{i}\right) \cdot H=\prod_{i=2}^{t} g_{i}$ is an element of $\widehat{S}$.

We can now establish the following Proposition 3.5:

Proposition 3.5: $\quad$ Let $\mathbb{L}=\mathbb{K}[a]$ be a finite extension of $\mathbb{I K}$ of degree $t$, provided with the unique absolute value extending that of $\mathbb{K}$ and let $a_{2}, \ldots, a_{t}$ be the conjugates of a over $\mathbb{K}$, with $a_{1}=a$. Let $\widehat{S}=\mathbb{L} \otimes_{\mathbb{K}} S$ and let $g \in \widehat{S}$ be such that $\inf _{\mathbb{E}}|g(x)|>0$. Then $g$ is invertible in $\widehat{S}$.

Proof: Let $g=\sum_{j=0}^{t} a^{j} f_{j}, f_{j} \in S$ and for every $k=1, \ldots, t$, let $g_{k}=\sum_{t}^{t}{ }_{j=0} a_{k}^{j} f_{j}, f_{j} \in S$. Then, by Proposition 3.4, $\prod_{k=1}^{t} g_{k}$ belongs to $S$ and in the same way, $\prod_{k=2}^{t} g_{k}$ belongs to $\widehat{S}$. Now, since $\inf _{\mathbb{E}}|g(x)|$ is a number $m>0$, we have $\left|\prod_{k=1}^{t} g_{k}\right| \geq m^{t}$ because in $\mathbb{L}$, we have $\left|g_{k}(x)\right|=\left|g_{1}(x)\right| \forall k=1,2, \ldots, t, \forall x \in \mathbb{E}$. Consequently, $\prod_{k=1}^{t} g_{k}$ is invertible in $S$. Thus, there exists $f \in S$ such that $\prod_{k=1}^{t} g_{k} . f=1$. But since, by Proposition 3.4, $\prod_{k=2}^{t} g_{k}$ belongs to $\widehat{S}$, one sees that $\prod_{k=2}^{t} g_{k} \cdot f$ is an element of $\widehat{S}$. Hence $g=g_{1}$ is invertible in $\widehat{S}$ with inverse $g^{-1}=\prod_{k=2}^{t} g_{k} \cdot f$ 
Definition and notation: In the following Proposition 3.7 and in the theorems we will have to consider the tensor product norm. We remind here some general facts (for completeness one can see [19]). Let $\mathbb{L}$ be a complete valued field extension of $\mathbb{K}$ and $A$ be a unital, ultrametric $\mathbb{K}$-Banach algebra. Given $z \in \mathbb{L} \otimes_{\mathbb{K}} A$, we put

$$
\|z\|_{\otimes}=\inf \left\{\max _{i \in I}\left|b_{i}\right| \cdot\left\|x_{i}\right\| \mid \sum_{i \in I} b_{i} \otimes_{\mathbb{K}} x_{i}=z, \text { Ifinite }\right\} .
$$

This norm $\|.\|_{\otimes}$ will be called the (projective) tensor product norm. It is an ultrametric norm.

In any unital $\mathbb{K}$-algebra $B$, let $1_{B}$ be the unity of $B$. Then for $b \in \mathbb{L}$ and $x \in B$, one has $\|b \otimes x\|_{\otimes}=|b| \cdot\|x\|$. In particular for any $b \in \mathbb{L}$, (resp. $x \in B$ ), one has $\left\|b \otimes 1_{B}\right\|_{\otimes}=|b|$ (resp. $\left\|1_{\mathbb{K}} \otimes x\right\|_{\otimes}=\|x\|$.) Hence one has an isometric identification of $\mathbb{L}$ (resp. $B$ ) with $\mathbb{L} \otimes_{\mathbb{K}} 1_{B}\left(\right.$ resp. $\left.1_{\mathbb{L}} \otimes_{\mathbb{K}} B\right)$.

Furthermore, one verifies that with the tensor norm, the tensor product $\mathbb{L} \otimes_{\mathbb{K}} B$, of the two unital $\mathbb{K}$-algebras $\mathbb{L}$ and $B$ is a normed unital $\mathbb{K}$-algebra. It is also a unital $\mathbb{L}$-algebra (obtained by extension of scalars). The completion $\mathbb{L} \widehat{\otimes_{\mathbb{K}}} B$ of $\mathbb{L} \otimes_{\mathbb{K}} B$ with respect to the tensor product norm $\|.\|_{\otimes}$ (called the topological tensor product) is a unital IK-Banach algebra as well as a $\mathbb{L}$-Banach algebra.

Now assume that $\mathbb{L}$ is of finite dimension $d$ over $\mathbb{K}$. Fix a $\mathbb{K}$-basis $\left(e_{j}\right)_{1 \leq j \leq d}$ of $\mathbb{L}$. It is readily seen that any $z \in \mathbb{L} \otimes_{\mathbb{K}} B$ can be written in the unique form $z=\sum_{j=1}^{d} e_{j} \otimes y_{j}$ and $\|z\|_{\otimes}=\left\|\sum_{j=1}^{d} e_{j} \otimes y_{j}\right\|_{\otimes} \leq \max _{1 \leq j \leq d}\left|e_{j}\right| \cdot\left\|y_{j}\right\|$.

On the other hand, given $b=\sum_{j=1}^{d} \beta_{j} e_{j} \in \mathbb{L}$, let us consider the norm $\|b\|_{1}=$ $\max _{1 \leq j \leq d}\left|\beta_{j}\right| \cdot\left|e_{j}\right|$. One has $|b| \leq\|b\|_{1}$ and since $\mathbb{L}$ is finite dimensional, there exists $\alpha>0$ such that $\alpha \max _{1 \leq j \leq d}\left|\beta_{j}\right| \cdot\left|e_{j}\right| \leq|b| \leq \max _{1 \leq j \leq d}\left|\beta_{j}\right| \cdot\left|e_{j}\right|$. Considering the dual basis $\left(e_{j}^{\prime}\right)_{1 \leq j \leq d}$ of $\left(e_{j}\right)_{1 \leq j \leq d}$ and the continuous linear operators $e_{j}^{\prime} \otimes i d_{B}$ of $\mathbb{L} \otimes_{\mathbb{K}} B$ into $\mathbb{K} \otimes_{\mathbb{K}} B=B$, one proves that $\alpha \max _{1 \leq j \leq d}\left|e_{j}\right| \cdot\left\|y_{j}\right\| \leq\|z\|_{\otimes} \leq \max _{1 \leq j \leq d}\left|e_{j}\right| \cdot\left\|y_{j}\right\|=\|z\|_{1}$.

This means that the norms $\|\cdot\|_{\otimes}$ and $\|\cdot\|_{1}$ of $\mathbb{L} \otimes_{\mathbb{K}} B$ are equivalent. One immediately sees that $\mathbb{L} \otimes_{\mathbb{K}} B$ equiped with the norm $\|z\|_{1}=\max _{1 \leq j \leq d}\left|e_{j}\right|\left\|y_{j}\right\|$ is isomorphic to the product $\mathbb{K}$-Banach space $B^{d}$ and then it is complete. It follows that $\left(\mathbb{L} \otimes_{\mathbb{K}} B,\|\cdot\|_{\otimes}\right)$ is complete and $\mathbb{L} \otimes_{\mathbb{K}} B=\mathbb{L} \widehat{\otimes_{\mathbb{K}}} B$.

One then has the following Theorem 3.6 contained in [19] (Chapter 4).

Theorem 3.6: If $\mathbb{L}$ is a finite extension of $\mathbb{K}$ and $B$ is a commutative unital $\mathbb{K}$ Banach algebra, then with the tensor product norm $\|.\|_{\otimes}$, the tensor product $\mathbb{L} \otimes_{\mathbb{K}} B$ of the $\mathbb{K}$-algebras $\mathbb{L}$ and $B$ is a $\mathbb{K}$-Banach algebra as well as a Banach algebra over $\mathbb{L}$.

Taking $B=S$, we can now conclude. 
Proposition 3.7 : $\quad$ Let $\mathbb{L}=\mathbb{K}[a]$ be a finite extension of $\mathbb{K}$ of degree $t$, provided with the unique absolute value extending that of $\mathbb{K}$. Then the algebra $\widehat{S}=\mathbb{L} \otimes_{\mathbb{K}} S$ provided with the tensor product norm \|\|$_{\otimes}$, is complete.

Moreover, $\widehat{S}$ can be identified with the Banach $\mathbb{L}$-algebra of functions $f$ from $\mathbb{E}$ to $\mathbb{L}$ of the form $f=\sum_{j=0}^{t-1} a^{j} f_{j}$ with $f_{j} \in S$ and $\widehat{S}$ is a semi-admissible $\mathbb{L}$-algebra.

Proof: By construction, $\widehat{S}$ is the set of functions $f=\sum_{j=0}^{t-1} a^{j} f_{j}$ with $f_{j} \in S$. Since each $f_{j}$ is continuous, so is $f$. By Theorem 3.6, $\widehat{S}$ is a Banach $\mathbb{L}$-algebra. Next, given a clopen subset $D$ of $\mathbb{E}$, the characteristic function $u$ of $D$ exists in $S$ and hence it belongs to $\widehat{S}$. Finally, given an element $g \in \widehat{S}$ such that $\inf _{x \in \mathbb{E}}|g(x)|>0$, by Proposition $3.5, g$ is invertible in $\widehat{S}$. Therefore, $\widehat{S}$ is semi-admissible.

Theorem 3.8: $\quad$ Let $\mathbb{L}$ be a finite extension of $\mathbb{I K}$ of degree $t$, provided with the unique absolute value extending that of $\mathbb{K}$ and let $\widehat{S}=\mathbb{L} \otimes_{\mathbb{K}} S$ be provided with the tensor product norm. Then $\widehat{S}$ is a semi-admissible Banach $\mathbb{L}$-algebra.

Proof: By definition, $\mathbb{L}$ is of the form $\mathbb{K}\left[b_{1}, \ldots, b_{q}\right]$ with $\mathbb{K}\left[b_{1}, \ldots, b_{j}\right]$ strictly included in $\mathbb{K}\left[b_{1}, \ldots, b_{j+1}\right], j=1, \ldots, q-1$. Put $\mathbb{L}_{j}=\mathbb{L}\left[b_{1}, \ldots, b_{j}\right], j=1, \ldots, q$ and $\widehat{S}_{j}=\mathbb{L}_{j} \otimes_{\mathbb{K}} S$. Suppose we have proved that $\widehat{S}_{j}$ is semi-admissible for some $j<q$. Next, since $\mathbb{L}_{j+1}=$ $\mathbb{L}_{j}\left[b_{j+1}\right]$, by Proposition 3.7, $\widehat{S}_{j+1}$ is semi-admissible. Therefore, by induction, $\widehat{S}_{q}=\widehat{S}$ is a semi-admissible Banach $\mathbb{L}$-algebra.

Theorem 3.9: $\quad$ Let $\mathcal{M}$ be a maximal ideal of finite codimension of $S$. Then $\mathcal{M}$ is of codimension 1.

Proof: $\quad$ Let $\mathbb{L}$ be the field $\frac{S}{\mathcal{M}}$ and let $\widehat{S}=\mathbb{L} \otimes_{\mathbb{K}} S$ be provided with the tensor product norm. By Theorem 3.8, $\widehat{S}$ is semi-admissible. Now, let $\chi$ be the morphism from $S$ onto $\mathbb{L}$ whose kernel is $\mathcal{M}$. Let $g \in S$ and let $b=\chi(g) \in \mathbb{L}$. By Theorem $3.5 \chi$ admits an extension to a morphism $\widehat{\chi}$ from $\widehat{S}$ to $\mathbb{L}$. But since $\widehat{S}$ is semi-admissible and since the kernel of $\widehat{\chi}$ is a maximal ideal $\widehat{\mathcal{M}}$ of $\widehat{S}$, by Theorem 2.1 there exists an ultrafilter $\mathcal{U}$ on $\mathbb{E}$ such that $\widehat{\mathcal{M}}=\mathcal{I}(\mathcal{U}, S)$. Take $g \in S$ and let $b=\chi(g)$. Then we have $\widehat{\chi}(g-b)=0$, hence $g-b$ belongs to $\widehat{\mathcal{M}}$, therefore $\lim _{\mathcal{U}} g(x)-b=0$ i.e. $\lim _{\mathcal{U}} g(x)=b$. But since $g \in S$, $g(x)$ belongs to $\mathbb{K}$ for all $x \in \mathbb{E}$. Therefore, since $\mathbb{K}$ is complete, $b$ belongs to $\mathbb{K}$. But by definition $\chi$ is a surjection from $S$ onto $\mathbb{L}$, hence every value $b$ of $\mathbb{L}$ actually lies in $\mathbb{K}$ and hence $\mathbb{L}=\mathbb{K}$.

\section{Multiplicative spectrum}

The multiplicative spectrum of a Banach $\mathbb{I K}$-algebra was first introduced by B. Guennebaud [16] and was at the basis of Berkovich's analytic space theory [2]. It was also much used in [12], [14], [15]. 
Notations and definitions: Let $T$ be a normed $\mathbb{K}$-algebra. We denote by $\operatorname{Mult}(T,\|\cdot\|)$ the set of continuous multiplicative algebra semi-norms of $T$ provided with the topology of pointwise convergence [3], which means that a basic neighborhood of some $\psi \in$ $\operatorname{Mult}(T, \|$. $\|)$ is a set of the form $W\left(\psi, f_{1}, \ldots, f_{q}, \epsilon\right)$, with $f_{j} \in T$ and $\epsilon>0$ and this is the set of $\phi \in \operatorname{Mult}(T,\|\|$.$) such that \left|\psi\left(f_{j}\right)-\phi\left(f_{j}\right)\right|_{\infty} \leq \epsilon \forall j=1, \ldots, q$. The topological space $\operatorname{Mult}(T,\|\|$.$) is then compact (see [2], or Theorem 6.2$ in [10]).

Given $\phi \in \operatorname{Mult}(T,\|\|$.$) , we call kernel of \phi$ the set of the $x \in T$ such that $\phi(x)=0$ and we denote it by $\operatorname{Ker}(\phi)$. It is a prime closed ideal of $T$ with respect to the norm $\|$. $\|$ $[10]$.

We denote by $\operatorname{Mult}_{m}(T,\|\|$.$) the set of continuous multiplicative semi-norms of T$ whose kernel is a maximal ideal and by $\operatorname{Mult}_{1}(T,\|\cdot\|)$ the set of continuous multiplicative semi-norms of $T$ whose kernel is a maximal ideal of codimension 1. Particularly, considering the algebra $S$, we denote by $\operatorname{Mult}_{\mathbb{E}}(S, \|$. \|) the set of continuous multiplicative semi-norms of $S$ whose kernel is a maximal ideal of the form $\mathcal{I}(a, S), a \in \mathbb{E}$.

We denote by $\Upsilon(T)$ the set of $\mathbb{K}$-algebra homomorphisms from $S$ to $\mathbb{K}$.

Let us recall that in $S$, we have $\|.\|_{0} \leq \|$. $\|_{s p}$ and that if $S$ is admissible, then $\|\cdot\|_{0}=\|\cdot\|_{s p}$. Theorem 4.1 is classical [10], [11]:

Theorem 4.1: Let $T$ be a unital commutative ultrametric Banach $\mathbb{K}$-algebra. For each $f \in T,\|f\|_{s p}=\sup \{\phi(f) \mid \phi \in \operatorname{Mult}(T,\|\|)$.$\} . For every \chi \in \Upsilon(T)$, we have $|\chi(f)| \leq$ $\|f\|_{s p} \forall f \in T$.

More notations: For any ultrafilter $\mathcal{U} \in U(\mathbb{E})$ and any $f \in S,|f(x)|$ has a limit along $\mathcal{U}$ since $f$ is bounded. Given $a \in \mathbb{E}$ we denote by $\varphi_{a}$ the mapping from $S$ to $\mathbb{R}$ defined by $\varphi_{a}(f)=|f(a)|$ and for any ultrafilter $\mathcal{U} \in U(\mathbb{E})$, we denote by $\varphi_{\mathcal{U}}$ the mapping from $S$ to $\mathbb{R}$ defined by $\varphi_{\mathcal{U}}(f)=\lim _{\mathcal{U}}|f(x)|$ (see Lemma 1.13). These maps belong to Mult $(S,\|$.$\| )$ since $\|\cdot\|_{0} \leq\|\cdot\|_{s p} \leq \|$. $\|$. Particularly, the elements of $\operatorname{Mult}_{\mathbb{E}}(S, \|$. \|) are the $\varphi_{a}, a \in \mathbb{E}$.

Proposition 4.2: Let $a \in \mathbb{E}$. Then $\mathcal{I}(a, S)$ is a maximal ideal of $S$ of codimension 1 and $\varphi_{a}$ belongs to $\operatorname{Mult}_{1}(S,\|\|$.$) . Conversely, for every algebra homomorophism \chi$ from $S$ to $\mathbb{K}$, its kernel is a maximal ideal of the form $\mathcal{I}(a, S)$ with $a \in \mathbb{E}$ and $\chi$ is defined as $\chi(f)=f(a)$, while $\varphi_{a}(f)=|\chi(f)|$.

Theorem 4.3: Let $\mathcal{U}$ be an ultrafilter on $\mathbb{E}$. Then $\varphi_{\mathcal{U}}$ belongs to the closure of $\operatorname{Mult}_{\mathbb{E}}(S,\|\|$.$) , with respect to the topology of \operatorname{Mult}(S,\|\|$.$) .$

Proof: Let $\psi=\varphi_{\mathcal{U}}$, take $\epsilon>0$ and let $f_{1}, \ldots, f_{q} \in S$. There exists $L \in \mathcal{U}$ such that $\left|\psi\left(f_{j}\right)-\right| f_{j}(x)||_{\infty} \leq \epsilon \forall x \in L, \forall j=1, \ldots, q$. Therefore, taking $a \in L$, we have $\mid \varphi_{a}\left(f_{j}\right)-$ $\left.\psi\left(f_{j}\right)\right|_{\infty} \leq \epsilon \forall j=1, \ldots, q$ which shows that $\varphi_{a}$ belongs to the neighborhood $W$ of $\psi$ of the form $\left\{\phi\left|\psi\left(f_{j}\right)-\phi\left(f_{j}\right)\right|_{\infty} \leq \epsilon \forall j=1, \ldots, q\right\}$ and this proves the claim.

Remark 5: In the field $\mathbb{K}$, we call monotonous distances sequence a sequence $\left(a_{n}\right)_{n \in \mathbb{N}}$ of $\mathbb{E}$ such that the sequence $\delta\left(a_{n}, a_{n+1}\right)_{n \in \mathbb{N}}$ is strictly monotonous. We call constant distances sequence a sequence $\left(a_{n}\right)_{n \in \mathbb{N}}$ of $\mathbb{E}$ such that $\delta\left(a_{n}, a_{m}\right)$ is constant when $n, m$ 
are big enough and $n \neq m$. According to Remark 3 , if $\mathbb{K}$ is locally compact and $\mathbb{E}$ admits monotonous distances sequences or constant distances sequences, we can define $\varphi_{\mathcal{U}} \in \operatorname{Mult}_{1}\left(S, \|\right.$. \|) which does not belong to $\operatorname{Mult}_{\mathbb{E}}(S,\|\|$.$) .$

Theorem 4.4: For each $\phi \in \operatorname{Mult}(S,\|\|),. \operatorname{Ker}(\phi)$ is a prime spectrally closed ideal.

Proof: Let $\phi \in \operatorname{Mult}(S, \|$. $\|)$ and let $f$ belong to the spectral closure of $\operatorname{Ker}(\phi)$. There exists a sequence $\left(f_{n}\right)_{n \in \mathbb{N}}$ of $\operatorname{Ker}(\phi)$ such that $\lim _{n \rightarrow \infty}\left\|f_{n}-f\right\|_{s p}=0$. By Theorem 4.1, since $\phi(g) \leq\|g\|_{s p} \forall g \in S$, we have $\lim _{n \rightarrow \infty} \phi\left(f_{n}-f\right)=0$. But $\phi\left(f_{n}\right)=0 \forall n \in \mathbb{N}$, hence it follows that $\phi(f)=\lim _{n \rightarrow+\infty} \phi\left(f_{n}-f\right)=0$. Therefore, $f$ belongs to $\operatorname{Ker}(\phi)$, which means that $\widetilde{\operatorname{Ker}(\phi)}=\operatorname{Ker}(\phi)$.

By Theorem 4.4 and Corollary 2.4.e, we have the following Corollary 4.4.a:

Corollary 4.4.a: Suppose $S$ is admissible. Then $\operatorname{Mult}(S, \|$. $\|)=\operatorname{Mult}_{m}(S, \|$. $\|)$.

Theorem 4.5 is classical (Theorem 6.15 in [10]).

Theorem 4.5: $\quad$ Let $T$ be a commutative unital ultrametric Banach $\mathbb{K}$-algebra. For every maximal ideal $\mathcal{M}$ of $T$, there exists $\phi \in \operatorname{Mult}_{m}(S,\|\|$.$) such that \mathcal{M}=\operatorname{Ker}(\phi)$.

Recall that a unital commutative Banach $\mathbb{K}$-algebra is said to be multbijective if every maximal ideal is the kernel of only one continuous multiplicative semi-norm.

Remark 6: There exist some rare cases of ultrametric Banach algebras that are not multbijective [8], [9].

Theorem 4.6: Suppose $S$ is admissible. Then $S$ is multbijective. Precisely if $\psi \in$ $\operatorname{Mult}(S, \|$. $\|)$ and $\operatorname{Ker}(\psi)=\mathcal{M}$ then $\psi=\varphi \mathcal{U}$ for every ultrafilter $\mathcal{U}$ such that $\mathcal{M}=$ $\mathcal{I}(\mathcal{U}, S)$.

Proof: Let $\psi \in \operatorname{Mult}_{m}(S, \|$. $\|)$, let $\mathcal{M}=\operatorname{Ker}(\psi)$ and $\mathcal{U}$ be an ultrafilter such that $\mathcal{M}=\mathcal{I}(\mathcal{U}, S)$.

Let $f \in S$. Notice that if $f \in \mathcal{M}$ then $\psi(f)=\varphi_{\mathcal{U}}(f)=0$. Now we assume that $f \notin \mathcal{M}$. So $\psi(f)$ and $\varphi_{\mathcal{U}}(f)$ are both strictly positive. We prove that they are equal.

First let $\epsilon>0$ and consider the set $L=\left\{x \in \mathbb{E}:|f(x)| \leq \varphi_{\mathcal{U}}(f)+\epsilon\right\}$. This set belongs to $\mathcal{U}$ and by Lemma 1.2, it is clopen. Therefore its characteristic function $u$ lies in $S$. We have $\varphi_{\mathcal{U}}(u)=1$. Consequently, we can derive that $\psi(u)=1$ because $u$ is idempotent and does not belong to $\mathcal{M}$. Therefore $\psi(u f)=\psi(f)$ and $\varphi_{\mathcal{U}}(u f)=\varphi_{\mathcal{U}}(f)$. By Theorem 4.1, we have $\psi(f)=\psi(u f) \leq\|u f\|_{s p}=\|u f\|_{0}$ because $S$ is admissible. But by definition of $L$ we have: $\|u f\|_{0} \leq \varphi_{\mathcal{U}}(f)+\epsilon$. Therefore, $\psi(f) \leq \varphi_{\mathcal{U}}(f)+\epsilon$. This holds for every $\epsilon>0$. Consequently we may conclude that $\psi(f) \leq \varphi_{\mathcal{U}}(f)$ for every $f \in S$.

We prove now the inverse inequality. We have $\varphi_{\mathcal{U}}(f)>0$, so consider the set $W=\{x \in$ $\left.\mathbb{E}:|f(x)| \geq \frac{\varphi \mathcal{U}(f)}{2}\right\}$. This is a clopen set which belongs to $\mathcal{U}$. Let $w$ be the characteristic function of $W$ and put $g=w f+(1-w)$. We have $\varphi_{\mathcal{U}}(w)=1$ and $\varphi_{\mathcal{U}}(1-w)=0$ so 
$w \notin \mathcal{M}$ and $1-w \in \mathcal{M}$. Since $\mathcal{M}=\operatorname{Ker}(\psi)$ we then have $\psi(1-w)=0$ and $\psi(w)=1$ because $w$ is idempotent. Finally $\psi(g)=\psi(f)$ and $\varphi_{\mathcal{U}}(g)=\varphi_{\mathcal{U}}(f)$.

On the other hand, we can check that $|g(x)| \geq \min \left(1, \frac{\varphi_{\mathcal{U}}(f)}{2}\right)$ for all $x \in \mathbb{E}$, hence $g$ is invertible in $S$. Putting $h=\frac{1}{g}$, using the first inequality yet proved, we have

$$
\psi(f)=\psi(g)=\frac{1}{\psi(h)} \geq \frac{1}{\varphi_{\mathcal{U}}(h)}=\varphi_{\mathcal{U}}(g)=\varphi_{\mathcal{U}}(f)
$$

That concludes the proof.

Remark 7: Thus, if $S$ is admissible, $\operatorname{Mult}(S, \|$. $\|)$ can be identified to $\operatorname{Mult}\left(S,\|\|_{0}\right)$

Corollary 4.6.a: Suppose $S$ is admissible. For every $\phi \in \operatorname{Mult}(S, \|$. $\|)$ there exists a unique $\mathcal{H} \in Y_{(\mathcal{R})}(\mathbb{E})$ such that $\phi(f)=\lim _{\mathcal{U}}|f(x)| \forall f \in S, \forall \mathcal{U} \in \mathcal{H}$.

Moreover, the mapping $\Psi$ that associates to each $\phi \in \operatorname{Mult}(S, \|$. $\|)$ the unique $\mathcal{H} \in$ $Y_{\mathcal{R}}(\mathbb{E})$ such that $\phi(f)=\lim _{\mathcal{U}}|f(x)| \forall f \in S, \forall \mathcal{U} \in \mathcal{H}$, is a bijection from Mult $(S, \|$. $\|)$ onto $Y_{(\mathcal{R})}(\mathbb{E})$.

Assuming that $S$ is admissible, since by Theorem 4.6 each element $\phi \in \operatorname{Mult}(S,\|\|$. is of the form $\varphi_{\mathcal{U}}$, Corollary 4.6.b is immediate from theorem 4.3:

Corollary 4.6.b: If $S$ is admissible, then $\operatorname{Mult}_{\mathbb{E}}(S, \|$. $\|)$ is dense in Mult $(S, \|$. $\|)$.

Theorem 4.7: The topological space $\mathbb{E}$, provided with its distance $\delta$, is homeomorphic to $\operatorname{Mult}_{\mathbb{E}}(S, \|$. $\|)$ provided with the restricted topology from that of $M$ ult $(S, \|$. $\|)$.

Proof: For every $a \in \mathbb{E}$, put $\Lambda(a)=\varphi_{a}$, take $f_{1}, \ldots, f_{q} \in S$ and $\epsilon>0$. We set $W^{\prime}\left(\varphi_{a}, f_{1}, \ldots, f_{q}, \epsilon\right)=W\left(\varphi_{a}, f_{1}, \ldots, f_{q}, \epsilon\right) \cap \operatorname{Mult}_{\mathbb{E}}(S, \|$. $\|)$. Considering the natural topology on $\mathbb{E}$, the filter of neighborhoods of $a$ admits for basis the family of disks $d_{\mathbb{E}}\left(a, r^{-}\right), r>$ 0 . We will show that it is induced through the mapping $\Lambda$ by the filter admitting for basis the family of neighborhoods of $\varphi_{a}$ in $\operatorname{Mult}(S, \|$. $\|)$. Indeed, take $\left.r \in\right] 0,1[$ and let $u$ be the characteristic function of $d_{\mathbb{E}}\left(a, r^{-}\right)$. Then $W^{\prime}\left(\varphi_{a}, u, r\right)$ is the set of $\varphi_{b}$ such that $\left|\varphi_{a}(u)-\varphi_{b}(u)\right|_{\infty}<\frac{1}{2}$, i.e. the $\Lambda(b)$ such that $b \in d_{\mathbb{E}}\left(a, r^{-}\right)$. Therefore the topology induced on $\mathbb{E}$ by $\operatorname{Mult}(S, \|$. $\|)$ is thinner than its metric topology.

Conversely, take some neighborhood $W^{\prime}\left(\varphi_{a}, f_{1}, \ldots, f_{q}, \epsilon\right)$ of $\varphi_{a}$ in $\operatorname{Mult}_{\mathbb{E}}(S, \|$. $\|)$. For each $j=1, \ldots, q$, the set of the $x \in \mathbb{E}$ such that $\left|\varphi_{x}\left(f_{j}\right)-\varphi_{a}\left(f_{j}\right)\right|_{\infty} \leq \epsilon$ is the set of the $x$ such that ||$f_{j}(x)|-| f_{j}(a)||_{\infty} \leq \epsilon$. But now, since each $f_{j}$ is continuous, the set of the $x$ such that ||$f_{j}(x)|-| f_{j}(a)||_{\infty} \leq \epsilon \forall j=1, \ldots, q$ is a neighborhood of $a$ in $\mathbb{E}$. Consequently, the metric topology of $\mathbb{E}$ is thinner than the topolgy induced by $\operatorname{Mult}_{\mathbb{E}}(S,\|\|$.$) and that$ finishes proving that the two topological spaces are homeomorphic.

Corollary 4.7.a: $\operatorname{Mult}(S, \|$. $\|)$ is a compactification of the topological space $\mathbb{E}$. 
Theorem 4.8: Let $\phi=\varphi_{\mathcal{U}} \in \operatorname{Mult}_{m}(S,\|\|$.$) , with \mathcal{U}$ an ultrafilter on $\mathbb{E}$, let $\Gamma$ be the field $\frac{S}{\operatorname{Ker}(\phi)}$ and let $\theta$ be the canonical surjection from $S$ onto $\Gamma$. Then, the mapping $\|$. $\|^{\prime}$ defined on $\Gamma$ by $\|\theta(f)\|^{\prime}=\phi(f) \forall f \in S$, is the quotient norm $\|$. $\|^{\prime}$ of $\|$. $\|_{0}$ defined on $\Gamma$ and is an absolute value on $\Gamma$. Moreover, if $\operatorname{Ker}(\phi)$ is of codimension 1 , then this absolute value is the one defined on $\mathbb{K}$ and coincides with the quotient norm of the norm $\|$. $\|$ of $S$.

Proof: Let $\mathcal{M}=\operatorname{Ker}\left(\varphi_{\mathcal{U}}\right)$. Let $t \in \Gamma$ and let $f \in S$ be such that $\theta(f)=t$. So, $\|t\|^{\prime} \geq \lim _{\mathcal{U}}|f(s)|$. Conversely, take $\epsilon>0$ and let $V=\left\{x \in \mathbb{E}:|f(x)| \leq \lim _{\mathcal{U}}|f(s)|+\epsilon\right\}$. By Lemma 1.2, the set $V$ is clopen and belongs to $\mathcal{U}$. The characteristic function $u$ of $\mathbb{E} \backslash V$ belongs to $\mathcal{M}$ and so does $u f$. But by construction, $(f-u f)(x)=0 \forall x \in \mathbb{E} \backslash V$ and $(f-u f)(x)=f(x) \forall x \in V$. Consequently, $\|f-u f\|_{0} \leq \lim _{\mathcal{U}}|f(s)|+\epsilon$ and therefore $\|t\|^{\prime} \leq\|f-u f\|_{0} \leq \lim _{\mathcal{U}}|f(s)|+\epsilon$. This finishes proving the equality $\|\theta(f)\|^{\prime}=\lim _{\mathcal{U}}|f(s)|$ and hence the mapping defined by $|\theta(f)|=\phi(f), f \in S$ is the quotient norm $\|$. $\|^{\prime}$ of $\|$. $\|_{0}$. Then it is multiplicative, hence it is an absolute value on $\Gamma$.

Now, suppose that $\mathcal{M}$ is of codimension 1 . Then $\Gamma$ is isomorphic to $\mathbb{K K}$ and its absolute value $\|$. $\|^{\prime}$ is continuous with respect to the topology of $\mathbb{K}$, hence it is equal to the absolute value of $\mathbb{K}$. Finally consider the quotient norm $\|.\|_{q}$ of the norm $\|$. $\|$ of $S$ : that quotient norm of course bounds the quotient norm $\|$. $\|^{\prime}$ which is the absolute value of $\mathbb{K}$. If $f \in S$ and $b=\theta(f)$, we have $f-b \in \mathcal{M}$ and $\|\theta(f)\|_{q} \leq\|b\|=|b|=|\theta(f)|=\|\theta(f)\|^{\prime}$, which ends the proof.

Corollary 4.8.a: Suppose that $S$ is admissible. Let $\phi \in \operatorname{Mult}(S, \|$. $\|)$, let $\Gamma$ be the field $\frac{S}{\operatorname{Ker}(\phi)}$ and let $\theta$ be the canonical surjection from $S$ onto $\Gamma$. Then, the mapping defined on $\Gamma$ by $|\theta(f)|=\phi(f), \forall f \in S$ is the quotient norm $\|$. $\|^{\prime}$ of $\|.\|_{0}$ on $\Gamma$ and is an absolute value on $\Gamma$. Moreover, if $\operatorname{Ker}(\phi)$ is of codimension 1, then this absolute value is the one defined on $\mathbb{K}$ and coincides with the quotient norm of the norm $\|$. \| of $S$.

Remark 9: It is not clear whether an algebra $S$ admits a prime closed ideal $\mathcal{P}$ (with respect to the norm $\|$.$\| ) which is not a maximal ideal. If it admits such a prime closed$ ideal, then it is not the kernel of a continuous multiplicative semi-norm. In such a case, the quotient algebra by $\mathcal{P}$ has no continuous absolute value extending that of $\mathbb{K}$, although it has no divisors of zero. Such a situation can happen in certain Banach algebras [4].

Definition and notation: Given a IK-normed algebra $G$, we call Shilov boundary of $G$ a closed subset $F$ of $\operatorname{Mult}(G, \|$. $\|)$ that is minimum with respect to inclusion, such that, for every $x \in G$, there exists $\phi \in F$ such that $\phi(x)=\|x\|_{s p}[12],[13]$.

Let us recall the following Theorem 4.9 given in [10] and [12]:

Theorem 4.9: Every normed $\mathbb{I K}$-algebra admits a Shilov boundary.

Notation: Given a normed $\mathbb{I K}$-algebra $G$, we denote by $\operatorname{Shil}(G)$ the Shilov boundary of $G$. 
Lemma 4.10: Let us fix $a \in \mathbb{E}$. For every $r>0$, let $Z(a, r)$ be the set of multiplicative semi-norms $\varphi_{\mathcal{U}}$, with $\mathcal{U} \in U(\mathbb{E})$, such that $d_{\mathbb{E}}(a, r)$ belongs to $\mathcal{U}$. The family $\{Z(a, r) \mid r \in$ ] $0,1[\}$ forms a basis of the filter of neighborhoods of $\varphi_{a}$.

Proof: Let $W\left(\varphi_{a}, f_{1}, \ldots, f_{q}, \epsilon\right)$ be a neighborhood of $\varphi_{a}$ in $\operatorname{Mult}(S, \|$. $\|)$. There exists $r>0$ such that, whenever $\delta(a, x) \leq r$ we have $\left|f_{j}(x)-f_{j}(a)\right| \leq \epsilon \forall j=1, \ldots, q$ and therefore, clearly, $\left|\varphi_{\mathcal{U}}\left(f_{j}\right)-\varphi_{a}\left(f_{j}\right)\right|_{\infty} \leq \epsilon \forall j=1, \ldots q$ for every $\mathcal{U}$ containing $d_{\mathbb{E}}(a, r)$. Thus $Z(a, r)$ is included in $W\left(\varphi_{a}, f_{1}, \ldots, f_{q}, \epsilon\right)$.

Conversely, consider a set $Z(a, r)$ with $r \in] 0,1$ [, let $u$ be the characteristic function of $d_{\mathbb{E}}(a, r)$ and consider $W\left(\varphi_{a}, u, r\right)$. Given $\psi=\varphi_{\mathcal{U}} \in W\left(\varphi_{a}, u, r\right)$, we have $\left|\psi(u)-\varphi_{a}(u)\right|_{\infty} \leq$ $r$. But $\left|\psi(u)-\varphi_{a}(u)\right|_{\infty}=|\psi(u)-1|_{\infty}=\left|\lim _{\mathcal{U}}\right| u(x)|-1|_{\infty}$. If $d_{\mathbb{E}}(a, r)$ belongs to $\mathcal{U}$, then $\lim _{\mathcal{U}}|u(x)|=1$ and therefore $\left|\lim _{\mathcal{U}}\right| u(x)|-1|_{\infty}=0$. But if $d_{\mathbb{E}}(a, r)$ does not belong to $\mathcal{U}$, then $\lim _{\mathcal{U}}|u(x)|=0$ and therefore $\left|\lim _{\mathcal{U}}\right| u(x)|-1|_{\infty}=1$. Consequently, since $r<1$, $W\left(\varphi_{a}, u, r\right)$ is included in $Z(a, r)$, which finishes proving that the family of $\left.Z(a, r), r \in\right] 0,1[$ is a basis of the filter of neighborhoods of $\varphi_{a}$.

Theorem 4.11: Suppose $S$ is admissible. The Shilov boundary of $S$ is equal to $\operatorname{Mult}(S,\|\cdot\|)$.

Proof: We will show that for every $a \in \mathbb{E}, \varphi_{a}$ belongs to $\operatorname{Shil}(S)$. So, let us fix $a \in \mathbb{E}$ and suppose that $\varphi_{a}$ does not belong to $\operatorname{Shil}(S)$. Since $\operatorname{Shil}(S)$ is a closed subset of $\operatorname{Mult}(S, \|$. $\|)$, there exists a neighborhood of $\varphi_{a}$ that contains no element of $\operatorname{Shil}(S)$. Therefore, by Lemma 4.10, there exists $s>0$ such that $Z(a, s)$ contains no element of $\operatorname{Shil}(S)$. Now, let $D=d_{\mathbb{E}}(a, s)$ and let $u$ be the characteristic function of $D$. Since any $\phi \in \operatorname{Mult}(S, \|$. $\|)$ satisfies either $\phi(u)=1$ or $\phi(u)=0$, there exists $\theta \in \operatorname{Shil}(S)$ such that $\theta(u)=\|u\|_{s p}=1$. Then, $\theta$ is of the form $\varphi_{\mathcal{U}}$, with $\mathcal{U} \in U(\mathbb{E})$ and $\mathcal{U}$ does not contain $D$. But since $u(x)=0 \forall x \in \mathbb{E} \backslash D$, we have $\theta(u)=0$, a contradiction. Consequently, for every $a \in \mathbb{E}, \varphi_{a}$ belongs to $\operatorname{Shil}(S)$ which is a closed subset of $\operatorname{Mult}(S, \|$. $\|)$ and since, by Corollary 4.6.b, $\operatorname{Mult}_{\mathbb{E}}(S, \|$. $\|)$ is dense in $\operatorname{Mult}(S,\|\|$.$) , then \operatorname{Shil}(S)$ is equal to $\operatorname{Mult}(S,\|\cdot\|)$.

\section{The Stone space of $\mathbb{B}(\mathbb{E})$.}

It was proved in [14] that for the algebra $\mathcal{A}$ of continuous bounded functions from $\mathbb{E}$ to $\mathbb{K}$, the Banaschewski compactification of $\mathbb{E}$ is homeomorphic to $\operatorname{Mult}\left(\mathcal{A},\|\cdot\|_{0}\right)$. Here we get the same result for admissible algebras.

We denote by $\mathbb{B}(\mathbb{E})$ the Boolean ring of clopen subsets of $\mathbb{E}$ provided with the laws $\Delta$ for the addition and $\cap$ for the multiplication. As usually called the Stone space of the Boolean ring $\mathbb{B}(\mathbb{E})$ is the space $\Sigma(\mathbb{E})$ of non-zero ring homomorphisms from $\mathbb{B}(\mathbb{E})$ onto $\mathbb{F}_{2}$, provided with the topology of pointwise convergence. This space is a compactification of $\mathbb{E}$ and is called the Banaschewski compactification of $\mathbb{E}$ (see for example [19] for further details). 
For every $\mathcal{U} \in U(\mathbb{E})$, we denote by $\zeta_{\mathcal{U}}$ the ring homomorphism from $\mathbb{B}(\mathbb{E})$ onto $\mathbb{F}_{2}$ defined by $\zeta_{\mathcal{U}}(O)=1$ for every $O \in \mathbb{B}(\mathbb{E})$ that belongs to $\mathcal{U}$ and $\zeta_{\mathcal{U}}(O)=0$ for every $O \in \mathbb{B}(\mathbb{E})$ that does not belong to $\mathcal{U}$.

Particularly, given $a \in \mathbb{E}$, we denote by $\zeta_{a}$ the ring homomorphism from $\mathbb{B}(\mathbb{E})$ onto $\mathbb{F}_{2}$ defined by $\zeta_{a}(O)=1$ for every $O \in \mathbb{B}(\mathbb{E})$ that contains $a$ and $\zeta_{a}(O)=0$ for every $O \in \mathbb{B}(\mathbb{E})$ that does not contain $a$.

\section{Throughout Paragraph 5, we suppose that $S$ is an admissible algebra.}

Remark 10: Let $\Sigma^{\prime}(\mathbb{E})$ be the set of $\zeta_{a}, a \in \mathbb{E}$. The mapping that associates $\zeta_{a} \in \mathbb{E}$ to $a \in \mathbb{E}$ defines a surjective mapping from $\mathbb{E}$ onto $\Sigma^{\prime}(\mathbb{E})$. That mapping is also injective because given $a, b \in \mathbb{E}$, there exists a clopen subset $F$ such that $a \in F$ and $b \notin F$.

By Corollary 4.6.a, we have a bijection $\Psi$ from $\operatorname{Mult}(S,\|\cdot\|)$ onto $Y_{(\mathcal{R})}(\mathbb{E})$ associating to each $\phi \in \operatorname{Mult}(S,\|\cdot\|)$ the unique $\mathcal{H} \in Y_{(\mathcal{R})}(\mathbb{E})$ such that $\phi(f)=\lim _{\mathcal{U}}|f(x)|, \mathcal{U} \in \mathcal{H}, f \in$ $S$, i.e. $\phi=\varphi_{\mathcal{U}}$ for every $\mathcal{U} \in \mathcal{H}$.

On the other hand, let us take some $\mathcal{H} \in Y_{(\mathcal{R})}(\mathbb{E})$ and ultrafilters $\mathcal{U}, \mathcal{V}$ in $\mathcal{H}$. Since $\mathcal{U}, \mathcal{V}$ own the same clopen subsets of $\mathbb{E}$, we have $\zeta_{\mathcal{U}}=\zeta_{\mathcal{V}}$ and hence we can define a mapping $\Xi$ from $Y_{\mathcal{R}}(\mathbb{E})$ into $\Sigma(E)$ which associates to each $\mathcal{H} \in Y_{\mathcal{R}}(\mathbb{E})$ the boolean homomorphism $\zeta_{\mathcal{U}}$ independant from $\mathcal{U} \in \mathcal{H}$

Lemma 5.1: $\Xi$ is a bijection from $Y_{\mathcal{R}}(\mathbb{E})$ onto $\Sigma(\mathbb{E})$.

Proof: Indeed, let $\mathcal{H}, \mathcal{K} \in Y_{(\mathcal{R})}(\mathbb{E})$ and suppose that $\mathcal{H} \neq \mathcal{K}$. Take ultrafilters $\mathcal{U} \in \mathcal{H}$ and $\mathcal{V} \in \mathcal{K}$. They are not sticked, therefore by Theorem 1.12, there exists clopens $L \in$ $\mathcal{H}, M \in \mathcal{K}$ such that $H \cap K=\emptyset$. Then, $\Xi(\mathcal{H}) \neq \Xi(\mathcal{K})$, which proves the injectivity.

Now, let us check that $\Xi$ is surjective. Let $\theta \in \Sigma(\mathbb{E})$. Since $\theta$ is a ring homomorphism for the Boolean laws, the family of clopen sets $X$ satisfying $\theta(X)=1$ generates a filter $\mathcal{F}$. Let $\mathcal{U} \in U(\mathbb{E})$ be thinner than $\mathcal{F}$ and let $\mathcal{H}$ be the class of $\mathcal{U}$ with respect to $(\mathcal{R})$. We will check that $\theta=\Xi(\mathcal{H})=\zeta_{\mathcal{U}}$. Let $O$ be a clopen subset that belongs to $\mathcal{U}$. Then $\mathbb{E} \backslash O$ does not belong to $\mathcal{U}$ and therefore it does not belong to $\mathcal{F}$, so $\theta(\mathbb{E} \backslash O)=0$, consequently $\theta(O)=1$. And now, let $O$ be a clopen subset that does not belong to $\mathcal{U}$. Then $O$ does not belong to $\mathcal{F}$, hence $\theta(O)=0$, which ends the proof.

We put $\Phi=\Xi \circ \Psi$ and hence $\Phi$ is a bijection from $\operatorname{Mult}(S,\|\cdot\|)$ onto $\Sigma(\mathbb{E})$. Notice that for every ultrafilter $\mathcal{U}, \Psi\left(\varphi_{\mathcal{U}}\right)$ is the class $\mathcal{H}$ of $\mathcal{U}$ with respect to $(\mathcal{R})$ and $\Xi(\mathcal{H})=\zeta_{\mathcal{U}}$ so $\Phi\left(\varphi_{\mathcal{U}}\right)=\zeta_{\mathcal{U}}$.

Theorem 5.2: $\Phi$ is a homeomorphism once $\Sigma(\mathbb{E})$ and $\operatorname{Mult}(S, \|$. $\|)$ are provided with topologies of pointwise convergence.

Proof: Recall that for any $\mathcal{U} \in U(\mathbb{E})$, a neighborhoods basis of $\varphi_{\mathcal{U}}$ in $\operatorname{Mult}(S, \|$. $\|)$ is given by the family of sets of the form $W\left(\varphi_{\mathcal{U}}, f_{1}, \ldots, f_{q}, \epsilon\right)$ with $f_{1}, \ldots, f_{q} \in S, \epsilon>0$ and

$$
W\left(\varphi_{\mathcal{U}}, f_{1}, \ldots, f_{q}, \epsilon\right)=\left\{\varphi_{\mathcal{V}}|| \lim _{\mathcal{U}}\left|f_{j}(x)\right|-\left.\lim _{\mathcal{V}}\left|f_{j}(x)\right|\right|_{\infty} \leq \epsilon, j=1, \ldots, q\right\}
$$


On the other hand, for any $\mathcal{U} \in U(\mathbb{E})$, a neighborhood basis for $\zeta_{\mathcal{U}}$ in $\Sigma(\mathbb{E})$ is given by the family of sets $V\left(\zeta_{\mathcal{U}}, O_{1}, \ldots, O_{q}\right)$ where $O_{1}, \ldots, O_{q}$ belong to $\mathrm{B}(E)$ and

$$
V\left(\zeta_{\mathcal{U}}, O_{1}, \ldots, O_{q}\right)=\left\{\zeta_{\mathcal{V}} \mid \zeta_{\mathcal{U}}\left(O_{j}\right)=\zeta_{\mathcal{V}}\left(O_{j}\right), j=1, \ldots, q\right\}
$$

Notice also that if $F$ belongs to $\mathbb{B}(\mathbb{E})$ and if $u$ is its characteristic function, then for any $\mathcal{U} \in U(\mathbb{E})$, we have $\zeta_{\mathcal{U}}(F)=1$ if and only if $F \in \mathcal{U}$, i.e. if and only if $\lim _{\mathcal{U}}|u(x)|=1$. Otherwise, both $\zeta_{\mathcal{U}}(F)$ and $\lim _{\mathcal{U}}|u(x)|$ are equal to 0 . Therefore, the relation

$$
\left|\lim _{\mathcal{U}}\right| u(x)\left|-\lim _{\mathcal{V}}\right| u(x)||_{\infty} \leq \frac{1}{2}
$$

holds if and only if $\zeta_{\mathcal{U}}(F)=\zeta_{\mathcal{V}}(F)$. Recall that for every $\mathcal{U} \in U(\mathbb{E})$ we have $\Phi\left(\varphi_{\mathcal{U}}\right)=\zeta_{\mathcal{U}}$.

We will show that $\Phi$ is continuous. Consider $O_{1}, \ldots, O_{q} \in \mathbb{B}(\mathbb{E}), \mathcal{U} \in U(\mathbb{E})$ and the neighborhood $V\left(\zeta_{\mathcal{U}}, O_{1}, \ldots, O_{q}\right)$ of $\zeta_{\mathcal{U}}$. From the preceding remark, $\zeta_{\mathcal{V}}$ belongs to $V\left(\zeta_{\mathcal{U}}, O_{1}, \ldots, O_{q}\right)$ if and only if for every $j=1, \ldots, q, \zeta_{\mathcal{U}}\left(O_{j}\right)=\zeta_{\mathcal{V}}\left(O_{j}\right)$, i.e. if for every $j=1, \ldots, q$,

$$
\left|\lim _{\mathcal{U}}\right| u_{j}(x)\left|-\lim _{\mathcal{V}}\right| u_{j}(x)||_{\infty} \leq \frac{1}{2}
$$

i.e. if $\varphi_{\mathcal{V}}$ belongs to $W\left(\varphi_{\mathcal{U}}, u_{1}, \ldots, u_{q}, \frac{1}{2}\right)$. Consequently, this proves that $\Phi$ is continuous. We can now deduce that $\Phi$ is a homeomorphism because it is a continuous bijection between compact spaces (Corollaire 2 of Theoreme 2 in [3]).

Corollary 5.2.a: The space $\Sigma(\mathbb{E})$ is a compactification of $\mathbb{E}$ which is equivalent to the compactification $\operatorname{Mult}(S, \|$. \|).

Remark 11: For an admissible algebra $S$, the Banaschewski compactification $\Sigma(\mathbb{E})$ coincides with the Guennebaud-Berkovich multiplicative spectrum.

\section{References:}

[1] Amice, Y. Les nombres p-adiques, P.U.F. (1975).

[2] Berkovich, V. Spectral Theory and Analytic Geometry over Non-archimedean Fields. AMS Surveys and Monographs 33, (1990).

[3] Bourbaki, N. Topologie générale, Ch.3. Actualités scientifiques et industrielles, Hermann, Paris.

[4] Boussaf, K. and Escassut, A. Absolute values on algebras of analytic elements, Annales Mathématiques Blaise Pascal 2, n2 (1995).

[5] Chicourrat, M, Diarra, B. and Escassut, A. Finite codimensional maximal ideals in subalgebras of ultrametric uniformly continuous functions. Bulletin of the Belgian Mathematical Society- Simon Stevin, Vol 26, 3, (2019). 
[6] Chicourrat, $\mathbf{M}$ and Escassut, A. Ultrafilters and ultrametric Banach algebras of Lipschitz functions, Advances in Operator Theory 5(1), p. 154-142 (2020).

[7] G. Choquet Sur les notions de filtre et de grille. C.R.A.S., 224, pp.171-173, (1947).

[8] Escassut, A. Spectre maximal d'une algèbre de Krasner. Colloquium Mathematicum (Wroclaw) XXXVIII2, pp 339-357 (1978).

[9] Escassut, A. Analytic elements in p-adic analysis. World Scientific Publishing Co (1995).

[10] Escassut, A. The ultrametric Banach algebras. World Scientific Publishing Co (2003).

[11] Escassut, A. and Maïnetti, N. Spectral semi-norm of a p-adic Banach algebra, Bulletin of the Belgian Mathematical Society, Simon Stevin, vol 8, p.79-61, (1998).

[12] Escassut, A. and Maïnetti, N. Shilov boundary for ultrametric algebras, p-adic Numbers in Number Theory, Analytic Geometry and Functional Analysis, Belgian Mathematical Society, p.81-89, (2002).

[13] Escassut, A. Topological divisors of zero and Shilov boundary. Topology and its Applications 153, p. 1152-1163 (2006).

[14] Escassut, A. and Maïnetti, N. Multiplicative spectrum of ultrametric Banach algebras of continuous functions. Topology and its applications 157, p. 2505-25015 (2010).

[15] Escassut, A. and Maïnetti, N. Spectrum of ultrametric Banach algebras of strictly differentiable functions. Contemporary Mathematics 704, p. 139-160 (2018).

[16] Guennebaud, B. Sur une notion de spectre pour les algèbres normées ultramétriques, thèse d'Etat, Université de Poitiers, (1973).

[17] Haddad, L. Sur quelques points de topologie générale. Théorie des nasses et des tramails. Annales de la Faculté des Sciences de Clermont N 44, fasc.7, p.3-80 (1972).

[18] Van der Put, M. The Non-Archimedean Corona Problem. Table Ronde Anal. non Archimedienne, Bull. Soc. Math. Mémoire 39-40, p. 287-317 (1974).

[19] Van Rooij, A. Non-Archimedean Functional Analysis. Marcel Dekker (1978).

Laboratoire de Mathématiques Blaise Pascal,

Université Clermont Auvergne CNRS UMR 6620

3 Place Vasarely

63178 AUBIERE-CEDEX

FRANCE 